\title{
An Algebraic Approach to the Evolution of Emittances upon Crossing the Linear Coupling Difference Resonance
}

\author{
C.J. Gardner
}

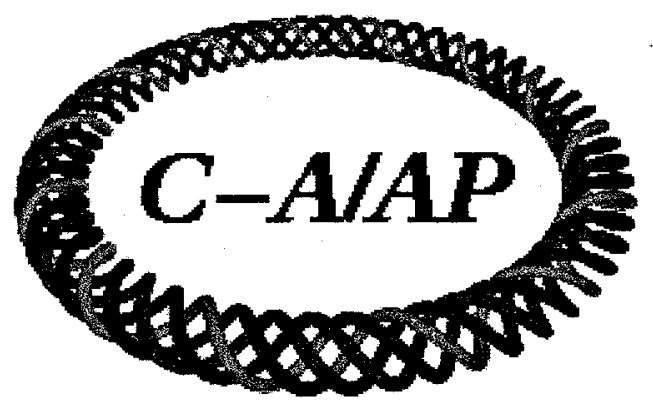

Collider-Accelerator Department Brookhaven National Laboratory

Upton, NY 11973

Notice: This document has been authorized by employees of Brookbaven Science Associates, LLC under Contract No. DE-AC02-98CH10886 with the U.S. Department of Energy. The United States Government retains a nonexclusive, paid-up, irrevocable, world-wide license to publish or reproduce the published form of this document, or allow others to do so, for United States Government purposes. 


\section{An Algebraic Approach to the Evolution of Emittances upon Crossing the Linear Coupling Difference Resonance

\author{
C.J. Gardner
}

September 16, 2008 


\section{Contents}

1 Introduction $\quad 6$

2 The One-Turn Matrix $\quad 7$

2.1 Edwards-Teng Parameterization . . . . . . . . . . . 8

2.2 The Normalized Coupling Matrix . . . . . . . . . . . . 9

3 The Linear Coupling Resonances $\quad 11$

3.1 Single Particle Motion and Normalized Coordinates . . . . . . 11

3.2 The Matched Ellipsoid . . . . . . . . . . . . . . . 13

3.3 Horizontal and Vertical Amplitudes . . . . . . . . . . . . . . . . . . . 13

3.4 The Sum and Difference of $J_{x}$ and $J_{y} \ldots \ldots \ldots \ldots$

3.5 Further Algebraic Reduction of the Amplitudes . . . . . . 17

3.6 The Difference Resonance . . . . . . . . . . . . . . . . 19

3.7 The Sum Resonance . . . . . . . . . . . . . . . . 21

4 Crossing the Difference Resonance 22

4.1 The TBT One-Turn Matrix . . . . . . . . . . . . . . 22

4.2 The TBT Beam Ellipsoid and its Projections . . . . . . . . 24

4.3 Normalized TBT Equations . . . . . . . . . . . . 26

4.4 Calculation of the TBT Emittances . . . . . . . . . 27

4.5 Approximate Expression for the TBT Emittances . . . . . . 28

4.6 Comparison with Other Formulae . . . . . . . . . 31

4.7 TBT Emittance Examples . . . . . . . . . . . . . . 32

4.8 Approximate Equations for Emittance Exchange . . . . . . . 34

4.9 Demonstration of Emittance Exchange . . . . . . . . . . . 39

4.10 Emittance Exchange Examples . . . . . . . . . . . . 39

5 Summary $\quad 40$

6 Appendix A 41 


\section{List of Figures}

1 Horizontal and vertical amplitudes $J_{x}$ and $J_{y}$ and their sum versus turn plotted in black, red and blue respectively. Here normalized coupling matrix parameters $A=0.6$ and $B=$ 0.06 . The small-amplitude high-frequency oscillations seen on all of the curves are due to the parameter $B$ being small but nonzero. . . . . . . . . . . . . . . . . 49

2 Amplitudes $J_{x}$ and $J_{y}$ and their sum obtained with normalized coupling matrix parameters $A=0.6$ and $B=0$. Here the high-frequency oscillations are gone and the sum is constant. 49

3 Amplitudes $J_{x}$ and $J_{y}$ and their difference plotted in black, red and green respectively. Here normalized coupling matrix parameters $A=0.05$ and $B=0.5$. The small-amplitude high-frequency oscillations seen on all of the curves are due to the parameter $A$ being small but nonzero. . . . . . . . 50

$4 \quad$ Normal-mode tunes $Q_{1}$ and $Q_{2}$ versus turn number $n$. Here the black curve is $Q_{\text {in }}$ and the red curve is $Q_{2 n}$. These tunes are given by equations (270-274) with $Q=0.25, \Delta Q_{\max }=$ $0.05, \Delta Q_{\min }=0.005$ and $2 N=4000 \ldots \ldots \ldots \ldots$

5 Parameters $U_{n}, T_{n}, d_{n}, e_{n}$ and $\xi_{n}$ plotted in black, red, blue, green and violet, respectively, for the tunes shown in Figure 4. Note that $T_{n}$ reaches zero at $n=N=2000$ but does not pass through zero. . . . . . . . . . . . . . 51

6 Exact horizontal and vertical emittances $\epsilon_{x n}$ and $\epsilon_{y n}$ plotted in black and red, respectively, for the tunes and parameters of Figures 4 and 5 . These were obtained by iteration of equation (201) with starting parameters $\epsilon_{x 0}=0.7, \epsilon_{y 0}=0.3, c_{0}=0$ and $h_{0}=0$. The emittances are not exchanged in this case.

- 7 Elements $c_{n}$ and $h_{n}$ of normalized beam ellipsoid matrix $\mathcal{E}_{n}$ plotted in green and blue, respectively, for the tunes and parameters of Figures 4 and $5 \ldots \ldots \ldots \ldots$. . . . . . . 52 
8 Approximate emittances $\epsilon_{x n}$ and $\epsilon_{y n}$ plotted in black and red, respectively, for the tunes and parameters of Figures 4 and 5 . These were obtained from equations $(253-256)$. . . . . . 52

9 Overlay of approximate on exact emittances from Figures 6 and 8 . The approximate emittances are shown in gold and blue. Note that there is good agreement up to turn 2000. . . 53

10 Difference between exact and approximate emittances from Figure 9. The horizontal and vertical differences (approximate minus exact) are shown in black and red respectively. . 53

11 Parameters $U_{n}, T_{n}, d_{n}, e_{n}$ and $\xi_{n}$ plotted in black, red, blue, green and violet, respectively, for the case in which $Q_{\max }$ is reduced from 0.05 to 0.025 . Comparing with Figure 5 we see that the adiabatic parameter $\xi_{n}$ is reduced by a factor of 2 . . 54

12 Exact emittances $\epsilon_{x n}$ and $\epsilon_{y n}$ plotted in black and red, respectively, for the case in which $Q_{\max }$ is reduced from 0.05 to $0.025 \ldots \ldots \ldots \ldots \ldots \ldots \ldots$

13 Overlay of approximate on exact emittances for the case in which $Q_{\max }$ is reduced from 0.05 to 0.025 . The approximate emittances are shown in gold and blue. Note the improved agreement between approximate and exact emittances. . . . . 55

14 Difference between exact and approximate emittances for the case in which $Q_{\max }$ is reduced from 0.05 to 0.025 . Comparing with Figure 10 we see that the differences have been reduced by a factor of $2 \ldots \ldots \ldots \ldots \ldots \ldots$

15 Normal-mode tunes $Q_{1 n}$ and $Q_{2 n}$ plotted in black and red respectively. These tunes are given by equations $(270-272)$ and (287-288) with $Q=0.25, \Delta Q_{\max }=0.05, \Delta Q_{\min }=0.005$ and $2 N=4000 \ldots \ldots \ldots \ldots \ldots \ldots$

16 Parameters $U_{n}, T_{n}, d_{n}, e_{n}$ and $\xi_{n}$ plotted in black, red, blue, green and violet, respectively, for the tunes shown in Figure 15. Note that $T_{n}$ is monotonically increasing and passes through zero at $n=N=2000 \ldots \ldots \ldots \ldots$ 
17 Exact horizontal and vertical emittances $\epsilon_{x n}$ and $\epsilon_{y n}$ plotted in black and red, respectively, for the tunes and parameters of Figures 15 and 16. These were obtained by iteration of equations (201) and (292) with starting parameters $\epsilon_{x 0}=0.7$, $\epsilon_{y 0}=0.3, c_{0}=0$ and $h_{0}=0$. The emittances are exchanged in this case. . . . . . . . . . . . . . 57

18 Elements $c_{n}$ and $h_{n}$ of normalized beam ellipsoid matrix $\mathcal{E}_{n}$ plotted in green and blue, respectively, for the tunes and parameters of Figures 15 and $16 . \ldots \ldots \ldots 57$

19 Approximate emittances $\epsilon_{x n}$ and $\epsilon_{y n}$ plotted in black and red, respectively, for the tunes and parameters of Figures 15 and 16. These were obtained from equations (253-256) and (338$339) \ldots \ldots \ldots \ldots \ldots \ldots \ldots \ldots$

20 Overlay of approximate on exact emittances from Figures 17 and 19. The approximate emittances are shown in gold and blue. Note that there is good agreement over the whole range of 4000 turns. . . . . . . . . . . . . . . . 58

21 Difference between exact and approximate emittances from Figure 20. The horizontal and vertical differences (approximate minus exact) are shown in black and red respectively. .

22 Exact horizontal and vertical emittances $\epsilon_{x n}$ and $\epsilon_{y n}$ plotted in black and red, respectively, for the same parameters as before but with $2 N=500$ turns. . . . . . . . . . 59

23 Overlay of approximate on exact emittances from Figure 22. The approximate emittances are shown in gold and blue. Note that there is good agreement up to about 200 turns; afterward the agreement is poor. . . . . . . . . 6 60

24 Difference between exact and approximate emittances from Figure 23. The horizontal and vertical differences (approximate minus exact) are shown in black and red respectively. . 


\section{Introduction}

One of the hallmarks of linear coupling is the resonant exchange of oscillation amplitude between the horizontal and vertical planes when the difference between the unperturbed tunes is close to an integer. The standard derivation of this phenomenon (known as the difference resonance) can be found, for example, in the classic papers of Guignard $[1,2]$. One starts with an uncoupled lattice and adds a linear perturbation that couples the two planes. The equations of motion are expressed in hamiltonian form. As the difference between the unperturbed tunes approaches an integer, one finds that the perturbing terms in the hamiltonian can be divided into terms that oscillate slowly and ones that oscillate rapidly. The rapidly oscillating terms are discarded or transformed to higher order with an appropriate canonical transformation. The resulting approximate hamiltonian gives equations of motion that clearly exhibit the exchange of oscillation amplitude between the two planes.

If, instead of the hamiltonian, one is given the four-by-four matrix for one turn around a synchrotron, then one has the complete solution for the turn-by-turn (TBT) motion. However, the conditions for the phenomenon of amplitude exchange are not obvious from a casual inspection of the matrix. These conditions and those that give rise to the related sum resonance are identified in this report. The identification is made using the well known formalism of Edwards and Teng [3, 4, 5] and, in particular, the normalized coupling matrix of Sagan and Rubin [6]. The formulae obtained are general in that no particular hamiltonian or coupling elements are assumed. The only assumptions are that the one-turn matrix is symplectic and that it has distinct eigenvalues on the unit circle in the complex plane.

Having identified the conditions of the one-turn matrix that give rise to the resonances, we focus on the difference resonance and apply the formulae to the evolution of the horizontal and vertical emittances of a beam distribution upon passing through the resonance. Exact and approximate expressions for the TBT evolution of the emittances are derived and applied to a number of examples. 


\section{The One-Turn Matrix}

We write the four-by-four matrix $\mathbf{T}$ for one turn around a circular accelerator as

$$
\mathbf{T}=\left(\begin{array}{cc}
\mathbf{M} & \mathbf{n} \\
\mathbf{m} & \mathbf{N}
\end{array}\right)
$$

where $\mathbf{M}, \mathbf{N}, \mathbf{m}$, and $\mathbf{n}$ are two-by-two matrices. The matrix $\mathbf{T}$ is symplectic and, as shown by Brown and Servranckx [7], is specified by 10 independent parameters. It follows from the symplectic condition that if $\lambda$ is an eigenvalue then so is $1 / \lambda[8,9]$. We shall assume that the four eigenvalues of $\mathbf{T}$ are distinct and that none of them is equal to 1 or -1 . One then has $\lambda_{1}, 1 / \lambda_{1}, \lambda_{2}$ and $1 / \lambda_{2}$ as the eigenvalues, with $\lambda_{1} \neq \lambda_{2}$. Since the elements of $\mathbf{T}$ are real, the complex conjugate of an eigenvalue is also an eigenvalue: We shall assume that the four distinct eigenvalues of $\mathbf{T}$ lie on the unit circle in the complex plane. We then have

$$
\lambda_{1}=e^{i \psi_{1}}, \quad \lambda_{2}=e^{i \psi_{2}}
$$

where $\psi_{1}$ and $\psi_{2}$ are real and $\cos \psi_{1} \neq \cos \psi_{2}$. The tunes associated with the eigenvalues are

$$
Q_{1}=\frac{\psi_{1}}{2 \pi}, \quad Q_{2}=\frac{\psi_{2}}{2 \pi}
$$

and, under our assumptions, neither $Q_{1}, 2 Q_{1}, Q_{2}, 2 Q_{2}, Q_{1}+Q_{2}$ nor $Q_{1}-Q_{2}$ is equal to an integer.

Under these conditions it is possible to write [10]

$$
\mathbf{T}=\mathcal{W U W}^{-1}
$$

where

$$
\begin{gathered}
\mathcal{U}=\left(\begin{array}{cc}
\mathcal{A} & 0 \\
0 & \mathcal{B}
\end{array}\right), \quad \mathbf{0}=\left(\begin{array}{cc}
0 & 0 \\
0 & 0
\end{array}\right) \\
\mathcal{A}=\left(\begin{array}{rr}
\cos \psi_{1} & \sin \psi_{1} \\
-\sin \psi_{1} & \cos \psi_{1}
\end{array}\right), \quad \mathcal{B}=\left(\begin{array}{rr}
\cos \psi_{2} & \sin \psi_{2} \\
-\sin \psi_{2} & \cos \psi_{2}
\end{array}\right)
\end{gathered}
$$

and $\mathcal{W}$ is a four-by-four symplectic matrix. 


\subsection{Edwards-Teng Parameterization}

As shown by Edwards and Teng [3], Billing [4] and Roser [5], the matrix $\mathcal{W}$ can be written in the form

$$
\mathcal{W}=\mathbf{R} \mathcal{N}
$$

where

$$
\begin{aligned}
& \mathbf{R}=\left(\begin{array}{cc}
d \mathbf{I} & \overline{\mathbf{W}} \\
-\mathbf{W} & d \mathbf{I}
\end{array}\right), \quad \mathcal{N}=\left(\begin{array}{cc}
\mathcal{F} & \mathbf{0} \\
\mathbf{0} & \mathcal{G}
\end{array}\right) \\
& \mathbf{I}=\left(\begin{array}{ll}
1 & 0 \\
0 & 1
\end{array}\right), \quad \mathbf{W}=\left(\begin{array}{ll}
W_{11} & W_{12} \\
W_{21} & W_{22}
\end{array}\right)
\end{aligned}
$$

and

$$
\mathcal{F}=\frac{1}{\sqrt{\beta_{1}}}\left(\begin{array}{rr}
\beta_{1} & 0 \\
-\alpha_{1} & 1
\end{array}\right), \quad \mathcal{G}=\frac{1}{\sqrt{\beta_{2}}}\left(\begin{array}{rr}
\beta_{2} & 0 \\
-\alpha_{2} & 1
\end{array}\right)
$$

Here and throughout the text we use a bar to denote the symplectic conjugate [8] of a two-by-two matrix. Thus

$$
\overline{\mathbf{W}}=\left(\begin{array}{rr}
W_{22} & -W_{12} \\
-W_{21} & W_{11}
\end{array}\right)
$$

and we have the properties

$$
\begin{gathered}
\mathbf{W} \overline{\mathbf{W}}=\overline{\mathbf{W}} \mathbf{W}=\left(W_{11} W_{22}-W_{12} W_{21}\right) \mathbf{I}=|\mathbf{W}| \mathbf{I}, \\
\mathbf{W}+\overline{\mathbf{W}}=\left(W_{11}+W_{22}\right) \mathbf{I}=(\operatorname{Tr} \mathbf{W}) \mathbf{I} .
\end{gathered}
$$

Since $\mathcal{W}$ and $\mathcal{N}$ are symplectic, $\mathbf{R}$ must be symplectic and it follows that

$$
|\mathbf{W}|=1-d^{2} .
$$

The one-turn matrix $\mathbf{T}$ is therefore given in terms of the 10 independent parameters $\alpha_{1}, \beta_{1}, \psi_{1}, \alpha_{2}, \beta_{2}, \psi_{2}, W_{11}, W_{12}, W_{21}$ and $W_{22}$.

To obtain the Edwards-Teng parameters in terms of the elements of $\mathbf{T}$ we write

$$
\mathbf{T}=\mathbf{R U R}^{-1}
$$

where

$$
\mathbf{U}=\mathcal{N U N}^{-1}=\left(\begin{array}{cc}
\mathbf{A} & \mathbf{0} \\
\mathbf{0} & \mathbf{B}
\end{array}\right)
$$


and

$$
\mathbf{A}=\mathcal{F} \mathcal{A} \mathcal{F}^{-1}, \quad \mathbf{B}=\mathcal{G B \mathcal { G } ^ { - 1 }}
$$

Carrying out the matrix multiplications one finds

$$
\begin{array}{ll}
\mathbf{M}=d^{2} \mathbf{A}+\overline{\mathbf{W}} \mathbf{B W}, & \mathbf{N}=d^{2} \mathbf{B}+\mathbf{W} \mathbf{A} \overline{\mathbf{W}}, \\
\mathbf{m}=d(\mathbf{B W}-\mathbf{W A}), & \mathbf{n}=d(\overline{\mathbf{W}} \mathbf{B}-\mathbf{A} \overline{\mathbf{W}}) .
\end{array}
$$

These equations can be inverted to obtain $\mathbf{A}, \mathbf{B}, \mathbf{W}$ and $d$ in terms of $\mathbf{M}$, $\mathbf{N}, \mathbf{m}$ and $\mathbf{n}$. One finds

$$
\mathbf{A}=\mathbf{M}-\frac{\mathbf{n W}}{d}, \quad \mathbf{B}=\mathbf{N}+\frac{\mathbf{W n}}{d^{3}}, \quad \frac{\mathbf{W}}{d}=-\frac{\mathbf{m}+\overline{\mathbf{n}}}{d^{2} U}
$$

where

$$
d^{2}=\frac{1}{2}+\frac{T}{2 U}, \quad T=\operatorname{Tr}(\mathbf{M}-\mathbf{N})
$$

and

$$
U=\operatorname{Tr}(\mathbf{A}-\mathbf{B})= \pm \sqrt{T^{2}+4|\mathbf{m}+\overline{\mathbf{n}}|} .
$$

Here, as discussed in [6], we choose the sign in front of the square root so that $U$ has the same sign as $T$. It then follows that $d^{2} \geq 1 / 2$. For the case $T=0$ we take $U>0$. We shall also always take $d>0$. With these choices the elements of $\mathbf{A}, \mathbf{B}$, and $\mathbf{W}$ are uniquely specified by those of $\mathbf{M}, \mathbf{N}, \mathbf{m}$ and $\mathbf{n}$. Note that if there are no magnetic elements that couple the horizontal and vertical planes of oscillation, the matrix elements of $\mathbf{m}$ and $\mathbf{n}$ are zero and $|\mathbf{m}+\overline{\mathbf{n}}|=0$. This gives $U=T$ and $d^{2}=1$.

\subsection{The Normalized Coupling Matrix}

Sagan and Rubin [6] have shown that it is useful to introduce the normalized matrix

$$
\widehat{\mathbf{R}}=\mathcal{N}^{-1} \mathbf{R} \mathcal{N}
$$

Carrying out the matrix multiplications we find that

$$
\widehat{\mathbf{R}}=\left(\begin{array}{cc}
d \mathbf{I} & \overline{\mathbf{w}} \\
-\mathbf{w} & d \mathbf{I}
\end{array}\right)
$$

where

$$
\mathbf{w}=\mathcal{G}^{-1} \mathbf{W} \mathcal{F}=\left(\begin{array}{ll}
w_{11} & w_{12} \\
w_{21} & w_{22}
\end{array}\right)
$$


and

$$
\mathbf{w} \overline{\mathbf{w}}=\mathbf{W} \overline{\mathbf{W}}=\left(1-d^{2}\right) \mathbf{I} .
$$

The matrix $\mathbf{w}$ is the normalized coupling matrix. It is now useful [6] to define parameters $A, B, \omega$, and $\psi$ such that

$$
\begin{aligned}
& 2 A \cos \omega=w_{11}+w_{22}, \quad 2 A \sin \omega=w_{12}-w_{21} \\
& 2 B \cos \psi=w_{11}-w_{22}, \quad 2 B \sin \psi=w_{12}+w_{21} .
\end{aligned}
$$

This shows that $\mathbf{w}$ can always be written in the form

$$
\mathbf{w}=A \Omega+B \Psi
$$

where

$$
\Omega=\left(\begin{array}{rr}
\cos \omega & \sin \omega \\
-\sin \omega & \cos \omega
\end{array}\right), \quad \Psi=\left(\begin{array}{rr}
\cos \psi & \sin \psi \\
\sin \psi & -\cos \psi
\end{array}\right)
$$

The matrix

$$
\mathcal{W}=\mathbf{R} \mathcal{N}=\mathcal{N} \widehat{\mathbf{R}}
$$

is then specified by the 8 parameters $\alpha_{1}, \beta_{1}, \alpha_{2}, \beta_{2}, A, B, \omega$ and $\psi$.

The matrices $\Omega$ and $\Psi$ have the properties

$$
\Omega \Omega^{\dagger}=\Omega^{\dagger} \Omega=\Psi \Psi^{\dagger}=\Psi^{\dagger} \Psi=\mathbf{I}
$$

and

$$
\bar{\Omega}=\Omega^{\dagger}, \quad \bar{\Psi}=-\Psi, \quad \Psi^{\dagger}=\Psi
$$

where here and throughout the text we use a dagger to denote the transpose of a matrix or vector. Thus

$$
\mathbf{w}^{\dagger}=A \Omega^{\dagger}+B \Psi, \quad \overline{\mathbf{w}}=A \Omega^{\dagger}-B \Psi
$$

and we see that

$$
\overline{\mathbf{w}}-\mathbf{w}^{\dagger}=0
$$

if and only if $B=0$. Similarly we have

$$
\overline{\mathbf{w}}+\mathbf{w}^{\dagger}=0
$$

if and only if $A=0$. In Sections 3.4-3.7, the conditions (35) and (36) will be seen to be associated with the linear coupling difference and sum resonances respectively. 
The matrices $\Omega$ and $\Psi$ have the additional commutation properties

$$
\Psi \Omega^{\dagger}=\Omega \Psi, \quad \Omega^{\dagger} \Psi=\Psi \Omega
$$

which give

$$
\mathbf{w} \overline{\mathbf{w}}=\left(A^{2}-B^{2}\right) \mathbf{I}
$$

and therefore

$$
|\mathbf{w}|=|\mathbf{W}|=1-d^{2}=A^{2}-B^{2}
$$

We also have

$$
\Psi \Omega+\bar{\Omega} \bar{\Psi}=\Psi \Omega-\Omega^{\dagger} \Psi=\mathbf{0}
$$

and therefore

$$
\operatorname{Tr}(\Psi \Omega)=0
$$

\section{The Linear Coupling Resonances}

\subsection{Single Particle Motion and Normalized Coordinates}

Let

$$
\mathbf{Z}=\left(\begin{array}{c}
\mathbf{X} \\
\mathbf{Y}
\end{array}\right), \quad \mathbf{X}=\left(\begin{array}{c}
X \\
X^{\prime}
\end{array}\right), \quad \mathbf{Y}=\left(\begin{array}{c}
Y \\
Y^{\prime}
\end{array}\right)
$$

and

$$
\mathbf{Z}_{0}=\left(\begin{array}{c}
\mathbf{X}_{0} \\
\mathbf{Y}_{0}
\end{array}\right), \quad \mathbf{X}_{0}=\left(\begin{array}{c}
X_{0} \\
X_{0}^{\prime}
\end{array}\right), \quad \mathbf{Y}_{0}=\left(\begin{array}{c}
Y_{0} \\
Y_{0}^{\prime}
\end{array}\right)
$$

where $X_{0}, X_{0}^{\prime}, Y_{0}, Y_{0}^{\prime}$ are the initial positions and angles of a beam particle and $X, X^{\prime}, Y, Y^{\prime}$ are the positions and angles after $n$ turns around the accelerator. Then

$$
\mathbf{Z}=\mathbf{T}^{n} \mathbf{Z}_{0}=\mathcal{W U}^{n} \mathcal{W}^{-1} \mathbf{Z}_{0}
$$

where $\mathbf{T}$ is given by (4). Here we see that it is natural to introduce normalized coordinates [11]

$$
\widehat{\mathbf{Z}}=\mathcal{W}^{-1} \mathbf{Z}, \quad \widehat{\mathbf{Z}}_{0}=\mathcal{W}^{-1} \mathbf{Z}_{0}
$$

where

$$
\widehat{\mathbf{Z}}=\left(\begin{array}{c}
\widehat{\mathbf{X}} \\
\widehat{\mathbf{Y}}
\end{array}\right), \quad \widehat{\mathbf{X}}=\left(\begin{array}{c}
\widehat{X} \\
\widehat{X}^{\prime}
\end{array}\right), \quad \widehat{\mathbf{Y}}=\left(\begin{array}{c}
\widehat{Y} \\
\widehat{Y}^{\prime}
\end{array}\right)
$$

and

$$
\widehat{\mathbf{Z}}_{0}=\left(\begin{array}{c}
\widehat{\mathbf{X}}_{0} \\
\widehat{\mathbf{Y}}_{0}
\end{array}\right), \quad \widehat{\mathbf{X}}_{0}=\left(\begin{array}{c}
\widehat{X}_{0} \\
\widehat{X}_{0}^{\prime}
\end{array}\right), \quad \widehat{\mathbf{Y}}_{0}=\left(\begin{array}{c}
\widehat{Y}_{0} \\
\widehat{Y}_{0}^{\prime}
\end{array}\right)
$$


We then have

$$
\begin{gathered}
\widehat{\mathbf{Z}}=\mathcal{U}^{n} \widehat{\mathbf{Z}}_{0} \\
\widehat{\mathbf{X}}=\mathcal{A}^{n} \widehat{\mathbf{X}}_{0}, \quad \widehat{\mathbf{Y}}=\mathcal{B}^{n} \widehat{\mathbf{Y}}_{0}
\end{gathered}
$$

and using the identities

$$
\left(\mathcal{A}^{n}\right)^{\dagger} \mathcal{A}^{n}=\mathbf{I}, \quad\left(\mathcal{B}^{n}\right)^{\dagger} \mathcal{B}^{n}=\mathbf{I}
$$

we have

$$
\widehat{X}^{2}+\widehat{X}^{\prime 2}=\widehat{\mathbf{X}}^{\dagger} \widehat{\mathbf{X}}=\widehat{\mathbf{X}}_{0}^{\dagger} \widehat{\mathbf{X}}_{0}=\widehat{X}_{0}^{2}+\widehat{X}_{0}^{\prime 2}=\epsilon_{1}
$$

and

$$
\widehat{Y}^{2}+\widehat{Y}^{\prime 2}=\widehat{\mathbf{Y}}^{\dagger} \widehat{\mathbf{Y}}=\widehat{\mathbf{Y}}_{0}^{\dagger} \widehat{\mathbf{Y}}_{0}=\widehat{Y}_{0}^{2}+\widehat{Y}_{0}^{\prime 2}=\epsilon_{2} .
$$

These equations define the normal mode emittances, $\epsilon_{1}$ and $\epsilon_{2}$, and show that they are conserved quantities. They also show that there exist real phases $\phi_{1}$ and $\phi_{2}$ such that

$$
\begin{array}{ll}
\widehat{X}_{0}=\sqrt{\epsilon_{1}} \cos \phi_{1}, & \widehat{X}_{0}^{\prime}=\sqrt{\epsilon_{1}} \sin \phi_{1} \\
\widehat{Y}_{0}=\sqrt{\epsilon_{2}} \cos \phi_{2}, & \widehat{Y}_{0}^{\prime}=\sqrt{\epsilon_{2}} \sin \phi_{2} .
\end{array}
$$

The four parameters $\epsilon_{1}, \epsilon_{2}, \phi_{1}$ and $\phi_{2}$ are initial condition parameters which either determine or are determined by $X_{0}, X_{0}^{\prime}, Y_{0}$ and $Y_{0}^{\prime}$ through equations (45-47) and (51-54).

Multiplying (45) by $\mathcal{W}$ from the left gives $\mathbf{Z}$ in terms of $\widehat{\mathbf{Z}}$. Then using (31) we have

$$
\mathbf{Z}=\mathcal{W} \widehat{\mathbf{Z}}=\mathbf{R} \mathcal{N} \widehat{\mathbf{Z}}=\mathcal{N} \widehat{\mathbf{R}} \widehat{\mathbf{Z}}
$$

and

$$
\begin{array}{r}
\mathbf{X}=d \mathcal{F} \widehat{\mathbf{X}}+\overline{\mathbf{W}} \mathcal{G} \widehat{\mathbf{Y}}=\mathcal{F}\{d \widehat{\mathbf{X}}+\overline{\mathbf{w}} \widehat{\mathbf{Y}}\} \\
\mathbf{Y}=-\mathbf{W} \mathcal{F} \widehat{\mathbf{X}}+d \mathcal{G} \widehat{\mathbf{Y}}=\mathcal{G}\{d \widehat{\mathbf{Y}}-\mathbf{w} \widehat{\mathbf{X}}\}
\end{array}
$$

Here we see that use of the normalized coupling matrix and normalized coordinates gives simple expressions when $\mathcal{F}$ is factored out of the horizontal motion and $\mathcal{G}$ is factored out of the vertical motion. 


\subsection{The Matched Ellipsoid}

Consider now the matrix

$$
\mathbf{E}=\mathcal{W} \mathcal{W}^{\dagger}
$$

Using (4) we have

$$
\mathbf{T E T} \mathbf{T}^{\dagger}=\mathcal{W} \mathcal{U}^{\dagger} \mathcal{W}^{\dagger}
$$

and since $\mathcal{U} \mathcal{U}^{\dagger}=\mathbf{I}$ we have

$$
\mathbf{T E} \mathbf{T}^{\dagger}=\mathbf{E} .
$$

By construction the matrix $\mathbf{E}$ is real, symmetric and positive definite. (A real symmetric matrix $\mathbf{E}$ is positive definite if and only if the quadratic form $\mathbf{Z}^{\dagger} \mathbf{E Z}>0$ for every vector $\mathbf{Z} \neq \mathbf{0}$.) It follows that the set of initial positions and angles $X_{0}, X_{0}^{\prime}, Y_{0}, Y_{0}^{\prime}$ defined by

$$
\mathbf{Z}_{0}^{\dagger} \mathbf{E}^{-1} \mathbf{Z}_{0}=\epsilon
$$

is a four dimensional ellipsoid. After $n$ turns around the machine we have

$$
\mathbf{Z}=\mathbf{T}^{n} \mathbf{Z}_{0}
$$

and

$$
\mathbf{Z}^{\dagger} \mathbf{E}^{-1} \mathbf{Z}=\mathbf{Z}_{0}^{\dagger}\left(\mathbf{T}^{\dagger}\right)^{n} \mathbf{E}^{-1} \mathbf{T}^{n} \mathbf{Z}_{0}
$$

But $\mathbf{T E} \mathbf{T}^{\dagger}=\mathbf{E}$ implies

$$
\mathbf{T}^{\dagger} \mathbf{E}^{-1} \mathbf{T}=\mathbf{E}^{-1}
$$

and therefore (by induction)

$$
\left(\mathbf{T}^{\dagger}\right)^{n} \mathbf{E}^{-1} \mathbf{T}^{n}=\mathbf{E}^{-1} .
$$

Thus

$$
\mathbf{Z}^{\dagger} \mathbf{E}^{-1} \mathbf{Z}=\mathbf{Z}_{0}^{\dagger} \mathbf{E}^{-1} \mathbf{Z}_{0}=\epsilon
$$

and we see that the particle positions and angles lie on the same ellipsoid after each turn. The ellipsoid is then said to be matched to the lattice.

\subsection{Horizontal and Vertical Amplitudes}

Partitioning the matched ellipsoid matrix into two-by-two matrices we have

$$
\mathbf{E}=\left(\begin{array}{ll}
\mathbf{F} & \mathbf{C} \\
\mathbf{C}^{\dagger} & \mathbf{G}
\end{array}\right)
$$


The projections of the matched ellipsoid onto the $X, X^{\prime}$ and $Y, Y^{\prime}$ planes are then the regions defined by [7]

$$
\left\{\mathbf{X}^{\dagger} \mathbf{F}^{-1} \mathbf{X}\right\} \leq \epsilon, \quad\left\{\mathbf{Y}^{\dagger} \mathbf{G}^{-1} \mathbf{Y}\right\} \leq \epsilon
$$

respectively. The borders of these regions are the ellipses defined by taking the equal signs in equations (68). This suggests that we define horizontal and vertical amplitudes

$$
J_{x}=F^{-1}\left\{\mathbf{X}^{\dagger} \mathbf{F}^{-1} \mathbf{X}\right\}=F\left\{\mathbf{X}^{\dagger} \overline{\mathbf{F}} \mathbf{X}\right\}
$$

and

$$
J_{y}=G^{-1}\left\{\mathbf{Y}^{\dagger} \mathbf{G}^{-1} \mathbf{Y}\right\}=G\left\{\mathbf{Y}^{\dagger} \overline{\mathbf{G}} \mathbf{Y}\right\}
$$

respectively, where

$$
F=|\mathbf{F}|^{-1 / 2}, \quad G=|\mathbf{G}|^{-1 / 2}
$$

and we have used the identities

$$
\mathbf{F}^{-1}=|\mathbf{F}|^{-1} \overline{\mathbf{F}}, \quad \mathbf{G}^{-1}=|\mathbf{G}|^{-1} \overline{\mathbf{G}} .
$$

Note that by construction the matrices $F \mathbf{F}$ and $G \mathbf{G}$ have unit determinant and are symmetric and positive definite. Thus we can write

$$
F \mathbf{F}=\left(\begin{array}{rr}
b_{x} & -a_{x} \\
-a_{x} & g_{x}
\end{array}\right), \quad G \mathbf{G}=\left(\begin{array}{rr}
b_{y} & -a_{y} \\
-a_{y} & g_{y}
\end{array}\right)
$$

and we find that $J_{x}$ and $J_{y}$ have the familiar Courant-Snyder forms

$$
\begin{gathered}
J_{x}=g_{x} X^{2}+2 a_{x} X X^{\prime}+b_{x} X^{\prime 2} \\
J_{y}=g_{y} Y^{2}+2 a_{y} Y Y^{\prime}+b_{y} Y^{\prime 2}
\end{gathered}
$$

where

$$
b_{x} g_{x}-a_{x}^{2}=1, \quad b_{y} g_{y}-a_{y}^{2}=1
$$

Using (31) in (58) we have

$$
\mathbf{E}=\mathcal{W} \mathcal{W}^{\dagger}=\mathcal{N} \widehat{\mathbf{R}} \widehat{\mathbf{R}}^{\dagger} \mathcal{N}^{\dagger}
$$

and carrying out the matrix multiplications one finds

$$
\mathbf{F}=\mathcal{F}\left\{d^{2} \mathbf{I}+\overline{\mathbf{w}} \overline{\mathbf{w}}^{\dagger}\right\} \mathcal{F}^{\dagger}
$$




$$
\mathbf{G}=\mathcal{G}\left\{d^{2} \mathbf{I}+\mathbf{w w}^{\dagger}\right\} \mathcal{G}^{\dagger}
$$

and

$$
\mathbf{C}=d \mathcal{F}\left\{\overline{\mathbf{w}}-\mathbf{w}^{\dagger}\right\} \mathcal{G}^{\dagger}
$$

Thus we have

$$
\begin{gathered}
\overline{\mathbf{F}}=\overline{\mathcal{F}}^{\dagger}\left\{d^{2} \mathbf{I}+\mathbf{w}^{\dagger} \mathbf{w}\right\} \overline{\mathcal{F}} \\
\overline{\mathbf{G}}=\overline{\mathcal{G}}^{\dagger}\left\{d^{2} \mathbf{I}+\overline{\mathbf{w}}^{\dagger} \overline{\mathbf{w}}\right\} \overline{\mathcal{G}} \\
\mathbf{F} \overline{\mathbf{F}}=\left\{d^{2} \mathbf{I}+\overline{\mathbf{w}} \overline{\mathbf{w}}^{\dagger}\right\}\left\{d^{2} \mathbf{I}+\mathbf{w}^{\dagger} \mathbf{w}\right\} \\
\mathbf{G} \overline{\mathbf{G}}=\left\{d^{2} \mathbf{I}+\mathbf{w w}^{\dagger}\right\}\left\{d^{2} \mathbf{I}+\overline{\mathbf{w}}^{\dagger} \overline{\mathbf{w}}\right\}
\end{gathered}
$$

and

$$
|\mathbf{F}|=|\mathbf{G}|=1-2 d^{2}\left(1-d^{2}\right)+d^{2} \operatorname{Tr}\left(\mathbf{w} \mathbf{w}^{\dagger}\right) .
$$

Using (56-57) and (81-82) in (69-70) we then have

$$
\begin{aligned}
& J_{x}=F\left\{d \widehat{\mathbf{X}}^{\dagger}+\widehat{\mathbf{Y}}^{\dagger} \overline{\mathbf{w}}^{\dagger}\right\}\left\{d^{2} \mathbf{I}+\mathbf{w}^{\dagger} \mathbf{w}\right\}\{d \widehat{\mathbf{X}}+\overline{\mathbf{w}} \widehat{\mathbf{Y}}\} \\
& J_{y}=G\left\{d \widehat{\mathbf{Y}}^{\dagger}-\widehat{\mathbf{X}}^{\dagger} \mathbf{w}^{\dagger}\right\}\left\{d^{2} \mathbf{I}+\overline{\mathbf{w}}^{\dagger} \overline{\mathbf{w}}\right\}\{d \widehat{\mathbf{Y}}-\mathbf{w} \widehat{\mathbf{X}}\}
\end{aligned}
$$

where

$$
F=G=\left\{1-2 d^{2}\left(1-d^{2}\right)+d^{2} \operatorname{Tr}\left(\mathbf{w} \mathbf{w}^{\dagger}\right)\right\}^{-1 / 2} .
$$

\subsection{The Sum and Difference of $J_{x}$ and $J_{y}$}

Carrying out the multiplications in (86) and (87) we obtain

$$
\begin{aligned}
J_{x} & =F\left\{d^{4} \epsilon_{1}+\left(1-d^{2}\right)^{2} \epsilon_{2}\right\} \\
& +F\left\{2 d^{3} \widehat{\mathbf{X}}^{\dagger} \overline{\mathbf{w}} \widehat{\mathbf{Y}}+2 d\left(1-d^{2}\right) \widehat{\mathbf{X}}^{\dagger} \mathbf{w}^{\dagger} \widehat{\mathbf{Y}}\right\} \\
& +F\left\{d^{2} \widehat{\mathbf{Y}}^{\dagger} \overline{\mathbf{w}}^{\dagger} \overline{\mathbf{w}} \widehat{\mathbf{Y}}+d^{2} \widehat{\mathbf{X}}^{\dagger} \mathbf{w}^{\dagger} \mathbf{w} \widehat{\mathbf{X}}\right\} \\
J_{y} & =F\left\{d^{4} \epsilon_{2}+\left(1-d^{2}\right)^{2} \epsilon_{1}\right\} \\
& -F\left\{2 d^{3} \widehat{\mathbf{X}}^{\dagger} \mathbf{w}^{\dagger} \widehat{\mathbf{Y}}+2 d\left(1-d^{2}\right) \widehat{\mathbf{X}}^{\dagger} \overline{\mathbf{w}} \widehat{\mathbf{Y}}\right\} \\
& +F\left\{d^{2} \widehat{\mathbf{Y}}^{\dagger} \overline{\mathbf{w}}^{\dagger} \overline{\mathbf{w}} \widehat{\mathbf{Y}}+d^{2} \widehat{\mathbf{X}}^{\dagger} \mathbf{w}^{\dagger} \mathbf{w} \widehat{\mathbf{X}}\right\}
\end{aligned}
$$




$$
\begin{aligned}
J_{x}+J_{y} & =F\left\{1-2 d^{2}\left(1-d^{2}\right)\right\}\left(\epsilon_{1}+\epsilon_{2}\right) \\
& +F\left\{2 d\left(2 d^{2}-1\right) \widehat{\mathbf{X}}^{\dagger}\left(\overline{\mathbf{w}}-\mathbf{w}^{\dagger}\right) \widehat{\mathbf{Y}}\right\} \\
& +2 F\left\{d^{2} \widehat{\mathbf{Y}}^{\dagger} \overline{\mathbf{w}}^{\dagger} \overline{\mathbf{w}} \widehat{\mathbf{Y}}+d^{2} \widehat{\mathbf{X}}^{\dagger} \mathbf{w}^{\dagger} \mathbf{w} \widehat{\mathbf{X}}\right\}
\end{aligned}
$$

and

$$
\begin{aligned}
J_{x}-J_{y} & =F\left\{2 d^{2}-1\right\}\left(\epsilon_{1}-\epsilon_{2}\right) \\
& +F\left\{2 d \widehat{\mathbf{X}}^{\dagger}\left(\overline{\mathbf{w}}+\mathbf{w}^{\dagger}\right) \widehat{\mathbf{Y}}\right\}
\end{aligned}
$$

Here we see that if

$$
\overline{\mathbf{w}}=\mathbf{w}^{\dagger}
$$

then

$$
\begin{gathered}
\overline{\mathbf{w}}^{\dagger} \overline{\mathbf{w}}=\mathbf{w} \overline{\mathbf{w}}=\left(1-d^{2}\right) \mathbf{I} \\
\mathbf{w}^{\dagger} \mathbf{w}=\overline{\mathbf{w}} \mathbf{w}=\left(1-d^{2}\right) \mathbf{I} \\
\operatorname{Tr}\left(\mathbf{w} \mathbf{w}^{\dagger}\right)=\operatorname{Tr}\left(\mathbf{w}^{\dagger} \mathbf{w}\right)=2\left(1-d^{2}\right) \\
F=1
\end{gathered}
$$

and we have

$$
J_{x}+J_{y}=\epsilon_{1}+\epsilon_{2}
$$

This is just the result obtained in the perturbation treatment of the linear coupling difference resonance.

Similarly, if

$$
\overline{\mathbf{w}}=-\mathbf{w}^{\dagger}
$$

then

$$
\begin{gathered}
\overline{\mathbf{w}}^{\dagger} \overline{\mathbf{w}}=-\mathbf{w} \overline{\mathbf{w}}=\left(d^{2}-1\right) \mathbf{I} \\
\mathbf{w}^{\dagger} \mathbf{w}=-\overline{\mathbf{w}} \mathbf{w}=\left(d^{2}-1\right) \mathbf{I} \\
\operatorname{Tr}\left(\mathbf{w} \mathbf{w}^{\dagger}\right)=\operatorname{Tr}\left(\mathbf{w}^{\dagger} \mathbf{w}\right)=2\left(d^{2}-1\right) \\
F=\left\{1+4 d^{2}\left(d^{2}-1\right)\right\}^{-1 / 2}=\left\{2 d^{2}-1\right\}^{-1}
\end{gathered}
$$

and we have

$$
J_{x}-J_{y}=\epsilon_{1}-\epsilon_{2}
$$

which is just the result obtained in the perturbation treatment of the linear coupling sum resonance. 


\subsection{Further Algebraic Reduction of the Amplitudes}

As shown in Section 2.2 we have

$$
\begin{aligned}
& \mathbf{w}=A \Omega+B \Psi, \quad \mathbf{w}^{\dagger}=A \Omega^{\dagger}+B \Psi \\
& \overline{\mathbf{w}}=A \Omega^{\dagger}-B \Psi, \quad \overline{\mathbf{w}}^{\dagger}=A \Omega-B \Psi
\end{aligned}
$$

which give

$$
\begin{gathered}
\widehat{\mathbf{X}}^{\dagger} \overline{\mathbf{w}} \widehat{\mathbf{Y}}=A\left\{\widehat{\mathbf{X}}^{\dagger} \Omega^{\dagger} \widehat{\mathbf{Y}}\right\}-B\left\{\widehat{\mathbf{X}}^{\dagger} \Psi \widehat{\mathbf{Y}}\right\} \\
\widehat{\mathbf{X}}^{\dagger} \mathbf{w}^{\dagger} \widehat{\mathbf{Y}}=A\left\{\widehat{\mathbf{X}}^{\dagger} \Omega^{\dagger} \widehat{\mathbf{Y}}\right\}+B\left\{\widehat{\mathbf{X}}^{\dagger} \Psi \widehat{\mathbf{Y}}\right\} \\
\overline{\mathbf{w}}^{\dagger} \overline{\mathbf{w}}=(A \Omega-B \Psi)\left(A \Omega^{\dagger}-B \Psi\right) \\
\overline{\mathbf{w}}^{\dagger} \overline{\mathbf{w}}=\left(A^{2}+B^{2}\right) \mathbf{I}-2 A B \Omega \Psi \\
\widehat{\mathbf{Y}}^{\dagger} \overline{\mathbf{w}}^{\dagger} \overline{\mathbf{w}} \widehat{\mathbf{Y}}=\left(A^{2}+B^{2}\right) \epsilon_{2}-2 A B\left\{\hat{\mathbf{Y}}^{\dagger} \Omega \Psi \widehat{\mathbf{Y}}\right\} \\
\mathbf{w}^{\dagger} \mathbf{w}=\left(A \Omega^{\dagger}+B \Psi\right)(A \Omega+B \Psi) \\
\mathbf{w}^{\dagger} \mathbf{w}=\left(A^{2}+B^{2}\right) \mathbf{I}+2 A B \Psi \Omega \\
\widehat{\mathbf{X}}^{\dagger} \mathbf{w}^{\dagger} \mathbf{w} \widehat{\mathbf{X}}=\left(A^{2}+B^{2}\right) \epsilon_{1}+2 A B\left\{\widehat{\mathbf{X}}^{\dagger} \Psi \Omega \widehat{\mathbf{X}}\right\}
\end{gathered}
$$

and, using (41),

$$
\operatorname{Tr}\left(\mathbf{w} \mathbf{w}^{\dagger}\right)=\operatorname{Tr}\left(\mathbf{w}^{\dagger} \mathbf{w}\right)=2\left(A^{2}+B^{2}\right) .
$$

Using these results in (89), (90) and (88) we obtain

$$
\begin{aligned}
J_{x} & =F\left\{d^{4} \epsilon_{1}+\left(1-d^{2}\right)^{2} \epsilon_{2}+d^{2}\left(A^{2}+B^{2}\right)\left(\epsilon_{1}+\epsilon_{2}\right)\right\} \\
& +2 d A F\left\{\widehat{\mathbf{X}}^{\dagger} \Omega^{\dagger} \widehat{\mathbf{Y}}\right\}+2 d\left(1-2 d^{2}\right) B F\left\{\widehat{\mathbf{X}}^{\dagger} \Psi \widehat{\mathbf{Y}}\right\} \\
& +2 d^{2} A B F\left\{\widehat{\mathbf{X}}^{\dagger} \Psi \Omega \widehat{\mathbf{X}}-\widehat{\mathbf{Y}}^{\dagger} \Omega \Psi \widehat{\mathbf{Y}}\right\}
\end{aligned}
$$

and

$$
\begin{aligned}
J_{y} & =F\left\{d^{4} \epsilon_{2}+\left(1-d^{2}\right)^{2} \epsilon_{1}+d^{2}\left(A^{2}+B^{2}\right)\left(\epsilon_{1}+\epsilon_{2}\right)\right\} \\
& -2 d A F\left\{\widehat{\mathbf{X}}^{\dagger} \Omega^{\dagger} \widehat{\mathbf{Y}}\right\}+2 d\left(1-2 d^{2}\right) B F\left\{\widehat{\mathbf{X}}^{\dagger} \Psi \widehat{\mathbf{Y}}\right\} \\
& +2 d^{2} A B F\left\{\widehat{\mathbf{X}}^{\dagger} \Psi \Omega \widehat{\mathbf{X}}-\widehat{\mathbf{Y}}^{\dagger} \Omega \Psi \widehat{\mathbf{Y}}\right\}
\end{aligned}
$$

where

$$
F=\left\{1-2 d^{2}\left(1-d^{2}\right)+2 d^{2}\left(A^{2}+B^{2}\right)\right\}^{-1 / 2} .
$$


To proceed further we compute

$$
\begin{gathered}
\widehat{\mathbf{X}}^{\dagger} \Omega^{\dagger} \widehat{\mathbf{Y}}=\widehat{\mathbf{X}}_{0}^{\dagger}\left(\mathcal{A}^{n}\right)^{\dagger} \Omega^{\dagger} \mathcal{B}^{n} \widehat{\mathbf{Y}}_{0}=\widehat{\mathbf{X}}_{0}^{\dagger} \Omega^{\dagger}\left(\mathcal{A}^{n}\right)^{\dagger} \mathcal{B}^{n} \widehat{\mathbf{Y}}_{0} \\
\widehat{\mathbf{X}}^{\dagger} \Psi \hat{\mathbf{Y}}=\widehat{\mathbf{X}}_{0}^{\dagger}\left(\mathcal{A}^{n}\right)^{\dagger} \Psi \mathcal{B}^{n} \widehat{\mathbf{Y}}_{0}=\widehat{\mathbf{X}}_{0}^{\dagger} \Psi \mathcal{A}^{n} \mathcal{B}^{n} \widehat{\mathbf{Y}}_{0} \\
\widehat{\mathbf{Y}}^{\dagger} \Omega \Psi \widehat{\mathbf{Y}}=\widehat{\mathbf{Y}}_{0}^{\dagger}\left(\mathcal{B}^{n}\right)^{\dagger} \Omega \Psi \mathcal{B}^{n} \widehat{\mathbf{Y}}_{0}=\widehat{\mathbf{Y}}_{0}^{\dagger} \Psi \Omega^{\dagger} \mathcal{B}^{2 n} \widehat{\mathbf{Y}}_{0} \\
\widehat{\mathbf{X}}^{\dagger} \Psi \Omega \widehat{\mathbf{X}}=\widehat{\mathbf{X}}_{0}^{\dagger}\left(\mathcal{A}^{n}\right)^{\dagger} \Psi \Omega \mathcal{A}^{n} \widehat{\mathbf{X}}_{0}=\widehat{\mathbf{X}}_{0}^{\dagger} \Psi \Omega \mathcal{A}^{2 n} \widehat{\mathbf{X}}_{0}
\end{gathered}
$$

where the components of $\widehat{\mathbf{X}}_{0}$ and $\widehat{\mathbf{Y}}_{0}$ are given by (53-54). We then have

$$
\begin{gathered}
\widehat{\mathbf{X}}^{\dagger} \Omega^{\dagger} \widehat{\mathbf{Y}}=\sqrt{\epsilon_{1} \epsilon_{2}} \cos \xi_{-} \\
\widehat{\mathbf{X}}^{\dagger} \Psi \widehat{\mathbf{Y}}=\sqrt{\epsilon_{1} \epsilon_{2}} \cos \xi_{+} \\
\widehat{\mathbf{Y}}^{\dagger} \Omega \Psi \widehat{\mathbf{Y}}=\epsilon_{2} \cos \zeta_{2} \\
\widehat{\mathbf{X}}^{\dagger} \Psi \Omega \widehat{\mathbf{X}}=\epsilon_{1} \cos \zeta_{1}
\end{gathered}
$$

where

$$
\begin{gathered}
\xi_{-}=n \psi_{1}-n \psi_{2}+\phi_{2}-\phi_{1}+\omega \\
\xi_{+}=n \psi_{1}+n \psi_{2}-\phi_{1}-\phi_{2}+\psi \\
\zeta_{2}=2 n \psi_{2}-2 \phi_{2}+\psi-\omega \\
\zeta_{1}=2 n \psi_{1}-2 \phi_{1}+\psi+\omega
\end{gathered}
$$

and

$$
\psi_{1}=2 \pi Q_{1}, \quad \psi_{2}=2 \pi Q_{2} .
$$

Thus the horizontal and vertical amplitudes become

$$
\begin{aligned}
J_{x} & =F\left\{d^{4} \epsilon_{1}+\left(1-d^{2}\right)^{2} \epsilon_{2}+d^{2}\left(A^{2}+B^{2}\right)\left(\epsilon_{1}+\epsilon_{2}\right)\right\} \\
& +2 d F \sqrt{\epsilon_{1} \epsilon_{2}}\left\{A \cos \xi_{-}+\left(1-2 d^{2}\right) B \cos \xi_{+}\right\} \\
& +2 d^{2} A B F\left\{\epsilon_{1} \cos \zeta_{1}-\epsilon_{2} \cos \zeta_{2}\right\}
\end{aligned}
$$

and

$$
\begin{aligned}
J_{y} & =F\left\{d^{4} \epsilon_{2}+\left(1-d^{2}\right)^{2} \epsilon_{1}+d^{2}\left(A^{2}+B^{2}\right)\left(\epsilon_{1}+\epsilon_{2}\right)\right\} \\
& -2 d F \sqrt{\epsilon_{1} \epsilon_{2}}\left\{A \cos \xi_{-}-\left(1-2 d^{2}\right) B \cos \xi_{+}\right\} \\
& +2 d^{2} A B F\left\{\epsilon_{1} \cos \zeta_{1}-\epsilon_{2} \cos \zeta_{2}\right\}
\end{aligned}
$$

where

$$
1-d^{2}=A^{2}-B^{2}
$$


and

$$
F=\left\{1-2 d^{2}\left(1-d^{2}\right)+2 d^{2}\left(A^{2}+B^{2}\right)\right\}^{-1 / 2} .
$$

These expressions are exact. They give the amplitudes of oscillation in the horizontal and vertical planes in terms of the initial condition parameters $\epsilon_{1}, \epsilon_{2}, \phi_{1}$ and $\phi_{2}$, and the parameters $d, A, B, \omega, \psi, Q_{1}$ and $Q_{2}$ of the one-turn matrix. The simple appearance of the parameters $A$ and $B$ in the expressions shows the utility of using the normalized coupling matrix.

Note that each amplitude contains frequencies $Q_{1}-Q_{2}, Q_{1}+Q_{2}, 2 Q_{1}$ and $2 Q_{2}$. If $B=0$, only frequency $Q_{1}-Q_{2}$ appears, while if $A=0$, only frequency $Q_{1}+Q_{2}$ appears. Note also that frequency $Q_{1}-Q_{2}$ is absent from the sum of the amplitudes and is the only frequency present in the difference. In the next two subsections we show that the conditions $B=0$ and $A=0$ give rise to the difference and sum resonances respectively.

\subsection{The Difference Resonance}

For the case $B=0$ we have

$$
A^{2}=1-d^{2}, \quad F=1
$$

and equations (132) and (133) become

$$
J_{x}=d^{2} \epsilon_{1}+\left(1-d^{2}\right) \epsilon_{2}+2\left\{d^{2}\left(1-d^{2}\right) \epsilon_{1} \epsilon_{2}\right\}^{1 / 2} \cos \xi_{-}
$$

and

$$
J_{y}=d^{2} \epsilon_{2}+\left(1-d^{2}\right) \epsilon_{1}-2\left\{d^{2}\left(1-d^{2}\right) \epsilon_{1} \epsilon_{2}\right\}^{1 / 2} \cos \xi_{-}
$$

where

$$
\xi_{-}=2 \pi n\left(Q_{1}-Q_{2}\right)+\phi_{2}-\phi_{1}+\omega
$$

Here we see that the only $n$ dependence in the expressions for $J_{x}$ and $J_{y}$ is in the terms containing $\cos \xi_{-}$which oscillate with frequency $Q_{1}-Q_{2}$.

Moreover we have

$$
J_{x}+J_{y}=\epsilon_{1}+\epsilon_{2} .
$$

These are just the properties of the linear coupling difference resonance.

Now, it follows from (21) and (22) that

$$
d^{2}\left(1-d^{2}\right)=\frac{U^{2}-T^{2}}{4 U^{2}}=\frac{|\mathbf{m}+\overline{\mathbf{n}}|}{T^{2}+4|\mathbf{m}+\overline{\mathbf{n}}|}
$$


and since

$$
1-d^{2}=A^{2}-B^{2}=A^{2}>0
$$

we must have

$$
|\mathbf{m}+\overline{\mathbf{n}}|>0
$$

Thus we may define

$$
K^{2}=|\mathbf{m}+\overline{\mathbf{n}}|
$$

and we have

$$
U^{2}=T^{2}+4 K^{2}
$$

ànd

$$
d^{2}\left(1-d^{2}\right)=\frac{K^{2}}{T^{2}+4 K^{2}}
$$

Substituting (146) into (137) and (138) we see that the oscillations of $J_{x}$ and $J_{y}$ are greatest when $T=0$ and go to zero as $K^{2}$ goes to zero. We also have

$$
d^{2}=\frac{1}{2}+\frac{1}{2}\left\{\frac{T^{2}}{T^{2}+4 K^{2}}\right\}^{1 / 2}
$$

which shows that $d^{2}$ goes to $1 / 2$ as $T$ goes to zero and goes to one as $K^{2}$ goes to zero. Note that the parameters $T$ and $K$ are analogous to the unperturbed tune separation and the coupling parameter in the perturbation treatment $[1,2]$ of the linear coupling resonance.

By specifying values for parameters $A, B, Q_{1}, Q_{2}$ we obtain values for $d^{2}$, $U^{2}, K^{2}$, and $T^{2}$. As an example of the difference resonance let us take

$$
A=0.6, \quad B=0.06, \quad Q_{1}=5.2364, \quad Q_{2}=4.2236 .
$$

We then have

$$
\begin{gathered}
d^{2}=1+B^{2}-A^{2}=0.6436 \\
U=2 \cos \left(2 \pi Q_{1}\right)-2 \cos \left(2 \pi Q_{2}\right)=-0.1595 \\
K^{2}=d^{2}\left(1-d^{2}\right) U^{2}=0.005838
\end{gathered}
$$

and

$$
T^{2}=U^{2}-4 K^{2}=0.002099 .
$$

Figure 1 shows a plot of $J_{x}, J_{y}$ and $J_{x}+J_{y}$ obtained with these values. Here we have taken $\epsilon_{1}=\epsilon_{2}=1, \phi_{1}=\phi_{2}=0$ and $\omega=\psi=\pi / 4$. As expected we see $J_{x}$ and $J_{y}$ oscillations characteristic of the difference resonance. The small-amplitude high-frequency oscillations seen on all of 
the curves are due to the parameter $B$ being small but nonzero. If we set $B=0$ and keep $A, Q_{1}, Q_{2}$ the same as before, we obtain the curves shown in Figure 2. Here we see that the high-frequency oscillations are gone and the sum $J_{x}+J_{y}$ is constant.

\subsection{The Sum Resonance}

For the case $A=0$ we have

$$
B^{2}=d^{2}-1, \quad F=\left\{2 d^{2}-1\right\}^{-1}
$$

and equations (132) and (133) become

$$
J_{x}=d^{2} \epsilon_{1}+\left(d^{2}-1\right) \epsilon_{2}-2\left\{d^{2}\left(d^{2}-1\right) \epsilon_{1} \epsilon_{2}\right\}^{1 / 2} \cos \xi_{+}
$$

and

$$
J_{y}=d^{2} \epsilon_{2}+\left(d^{2}-1\right) \epsilon_{1}-2\left\{d^{2}\left(d^{2}-1\right) \epsilon_{1} \epsilon_{2}\right\}^{1 / 2} \cos \xi_{+}
$$

where

$$
\xi_{+}=2 \pi n\left(Q_{1}+Q_{2}\right)-\phi_{1}-\phi_{2}+\psi
$$

Here we see that the only $n$ dependence in the expressions for $J_{x}$ and $J_{y}$ is in the terms containing $\cos \xi_{+}$which oscillate with frequency $Q_{1}+Q_{2}$. Moreover we have

$$
J_{x}-J_{y}=\epsilon_{1}-\epsilon_{2} .
$$

These are just the properties of the linear coupling sum resonance.

Now since

$$
d^{2}-1=B^{2}-A^{2}=B^{2}>0
$$

it follows from (141) that we must have

$$
|\mathbf{m}+\overline{\mathbf{n}}|<0
$$

Thus we may define

$$
K^{2}=-|\mathbf{m}+\overline{\mathbf{n}}|
$$

and we have

$$
d^{2}\left(d^{2}-1\right)=\frac{K^{2}}{T^{2}-4 K^{2}}
$$


Substituting (161) into (154) and (155) we see in this case that the oscillations of $J_{x}$ and $J_{y}$ increase without bound as $T^{2}$ approaches $4 K^{2}$. The motion is unstable for $T^{2} \leq 4 K^{2}$. We also have

$$
d^{2}=\frac{1}{2}+\frac{1}{2}\left\{\frac{T^{2}}{T^{2}-4 K^{2}}\right\}^{1 / 2}
$$

which shows that $d^{2} \geq 1$ and $d^{2}$ goes to one as $K^{2}$ goes to zero.

As an example of the sum resonance let us take

$$
A=0.05, \quad B=0.50, \quad Q_{1}=5.2124, \quad Q_{2}=4.7973 .
$$

We then we have

$$
\begin{gathered}
d^{2}=1+B^{2}-A^{2}=1.2475 \\
U=2 \cos \left(2 \pi Q_{1}\right)-2 \cos \left(2 \pi Q_{2}\right)=-0.1176 \\
K^{2}=d^{2}\left(d^{2}-1\right) U^{2}=0.004268
\end{gathered}
$$

and

$$
T^{2}=U^{2}-4 K^{2}=0.03089
$$

Figure 3 shows a plot of $J_{x}, J_{y}$ and $J_{x}-J_{y}$ obtained with these values. Here we have taken $\epsilon_{1}=1, \epsilon_{2}=0.5, \phi_{1}=\phi_{2}=0$ and $\omega=\psi=\pi / 4$. As expected we see $J_{x}$ and $J_{y}$ oscillations characteristic of the sum resonance. The small-amplitude high-frequency oscillations seen on all of the curves are due to the parameter $A$ being small but nonzero.

\section{Crossing the Difference Resonance}

Having established that the condition $B=0$ is associated with the difference resonance, we use matrices that satisfy this condition to study the TBT evolution of a distribution of beam particles under the influence of the resonance.

\subsection{The TBT One-Turn Matrix}

Let us suppose that the one-turn matrix depends on the turn number $n$. We use a subscript $n$ to denote the dependence. The one-turn matrix after $n$ turns is then

$$
\mathbf{T}_{n}=\mathcal{W}_{n} \mathcal{U}_{n} \mathcal{W}_{n}^{-1}
$$


where the matrix $\mathcal{U}_{n}$ is specified by parameters

$$
\psi_{1 n}=2 \pi Q_{1 n}, \quad \psi_{2 n}=2 \pi Q_{2 n}
$$

and the matrix

$$
\mathcal{W}_{n}=\mathbf{R}_{n} \mathcal{N}_{n}=\mathcal{N}_{n} \widehat{\mathbf{R}}_{n}
$$

is specified by parameters $\alpha_{1 n}, \beta_{1 n}, \alpha_{2 n}, \beta_{2 n}, A_{n}, B_{n}, \omega_{n}$ and $\psi_{n}$. We consider one-turn matrices for which $B_{n}=0$. We assume that the phase $\omega_{n}$ appearing in the matrix $\widehat{\mathbf{R}}_{n}$ is independent of $n$ and has value $\omega$. We assume further that the Courant-Snyder parameters $\alpha_{1 n}, \beta_{1 n}, \alpha_{2 n}, \beta_{2 n}$ appearing in the matrix $\mathcal{N}_{n}$ are independent of $n$ and have values $\alpha_{1}, \beta_{1}$, $\alpha_{2}$, and $\beta_{2}$ respectively. These simplifying assumptions allow for tractable algebraic expressions while retaining the essential features of the TBT evolution of the beam distribution. The only $n$ dependence in the matrix $\mathcal{W}_{n}$ is then through the parameter

$$
e_{n}=A_{n}= \pm \sqrt{1-d_{n}^{2}}
$$

Thus we have

$$
\mathbf{T}_{n}=\mathcal{N} \widehat{\mathbf{R}}_{n} \mathcal{U}_{n} \widehat{\mathbf{R}}_{n}^{-1} \mathcal{N}^{-1}
$$

where

$$
\begin{gathered}
\widehat{\mathbf{R}}_{n}=\left(\begin{array}{cc}
d_{n} \mathbf{I} & e_{n} \Omega^{\dagger} \\
-e_{n} \Omega & d_{n} \mathbf{I}
\end{array}\right), \quad \mathcal{U}_{n}=\left(\begin{array}{cc}
\mathcal{A}_{n} & \mathbf{0} \\
\mathbf{0} & \mathcal{B}_{n}
\end{array}\right) \\
\mathcal{A}_{n}=\left(\begin{array}{rr}
\cos \psi_{1 n} & \sin \psi_{1 n} \\
-\sin \psi_{1 n} & \cos \psi_{1 n}
\end{array}\right), \quad \mathcal{B}_{n}=\left(\begin{array}{rr}
\cos \psi_{2 n} & \sin \psi_{2 n} \\
-\sin \psi_{2 n} & \cos \psi_{2 n}
\end{array}\right) \\
\mathcal{N}=\left(\begin{array}{ll}
\mathcal{F} & \mathbf{0} \\
\mathbf{0} & \mathcal{G}
\end{array}\right), \quad \Omega=\left(\begin{array}{rr}
\cos \omega & \sin \omega \\
-\sin \omega & \cos \omega
\end{array}\right)
\end{gathered}
$$

and

$$
\mathcal{F}=\frac{1}{\sqrt{\beta_{1}}}\left(\begin{array}{rr}
\beta_{1} & 0 \\
-\alpha_{1} & 1
\end{array}\right), \quad \mathcal{G}=\frac{1}{\sqrt{\beta_{2}}}\left(\begin{array}{rr}
\beta_{2} & 0 \\
-\alpha_{2} & 1
\end{array}\right)
$$

Partitioning $\mathbf{T}_{n}$ into two-by-two matrices we then have

$$
\mathbf{T}_{n}=\left(\begin{array}{cc}
\mathbf{M}_{n} & \mathbf{n}_{n} \\
\mathbf{m}_{n} & \mathbf{N}_{n}
\end{array}\right)
$$

where

$$
\mathbf{M}_{n}=\mathcal{F}\left\{d_{n}^{2} \mathcal{A}_{n}+e_{n}^{2} \mathcal{B}_{n}\right\} \mathcal{F}^{-1}
$$




$$
\begin{gathered}
\mathbf{N}_{n}=\mathcal{G}\left\{d_{n}^{2} \mathcal{B}_{n}+e_{n}^{2} \mathcal{A}_{n}\right\} \mathcal{G}^{-1} \\
\mathbf{m}_{n}=d_{n} e_{n} \mathcal{G}\left\{\mathcal{B}_{n} \Omega-\Omega \mathcal{A}_{n}\right\} \mathcal{F}^{-1} \\
\mathbf{n}_{n}=d_{n} e_{n} \mathcal{F}\left\{\Omega^{\dagger} \mathcal{B}_{n}-\mathcal{A}_{n} \Omega^{\dagger}\right\} \mathcal{G}^{-1}
\end{gathered}
$$

Now let

$$
\begin{gathered}
U_{n}=\operatorname{Tr}\left(\mathcal{A}_{n}-\mathcal{B}_{n}\right)=2 \cos \left(2 \pi Q_{1 n}\right)-2 \cos \left(2 \pi Q_{2 n}\right) \\
T_{n}=\operatorname{Tr}\left(\mathbf{M}_{n}-\mathbf{N}_{n}\right)=\left(2 d_{n}^{2}-1\right) U_{n}
\end{gathered}
$$

and

$$
K_{n}^{2}=\left|\mathbf{m}_{n}+\overline{\mathbf{n}}_{n}\right|
$$

Then we have

$$
U_{n}^{2}=T_{n}^{2}+4 K_{n}^{2}
$$

and

$$
d_{n}^{2}=\frac{1}{2}+\frac{T_{n}}{2 U_{n}}
$$

where the mode labeling ( 1 or 2 ) of the tunes is chosen so that $T_{n}$ and $U_{n}$ have the same sign. We shall specify $Q_{1 n}, Q_{2 n}$ and $K_{n}$ and then compute $U_{n}, T_{n}, d_{n}$ and $e_{n}$ for each $n$. Resonance crossing occurs when $T_{n}$ goes through zero. Here $U_{n}$ must change sign and this means that $\mathcal{A}_{n}$ and $\mathcal{B}_{n}$ must be interchanged in (178-181). Since

$$
d_{n}^{2}=e_{n}^{2}=\frac{1}{2}
$$

when $T_{n}=0$, we see that the elements of $\mathbf{M}_{n}$ and $\mathbf{N}_{n}$ are unchanged by the interchange of $\mathcal{A}_{n}$ and $\mathcal{B}_{n}$, but those of $\mathbf{m}_{n}$ and $\mathbf{n}_{n}$ change sign unless $e_{n}$ changes sign. (Note that $\Omega$ and $\Omega^{\dagger}$ commute with $\mathcal{A}_{n}$ and $\mathcal{B}_{n}$.) We shall take $e_{n} \geq 0$ for $T_{n} \leq 0$ and $e_{n} \leq 0$ for $T_{n}>0$. This then guarantees that the elements of $\mathbf{M}_{n}, \mathbf{N}_{n}, \mathbf{m}_{n}, \mathbf{n}_{n}$ are continuous as $T_{n}$ passes through zero.

\subsection{The TBT Beam Ellipsoid and its Projections}

Rather than track individual particles of a distribution we track the parameters of the ellipsoid containing the distribution. We assume that the initial beam distribution is contained inside the beam ellipsoid defined by

$$
\mathbf{Z}_{0}^{\dagger} \mathbf{E}_{0}^{-1} \mathbf{Z}_{0}=\epsilon
$$


where $\mathbf{E}_{0}$ is a four-by-four symmetric positive-definite matrix with unit determinant. After $n$ turns the beam distribution is contained inside the ellipsoid

$$
\mathbf{Z}_{n}^{\dagger} \mathbf{E}_{n}^{-1} \mathbf{Z}_{n}=\epsilon
$$

where $\mathbf{Z}_{n}$ and $\mathbf{E}_{n}$ are given by the recursion relations

$$
\mathbf{Z}_{n+1}=\mathbf{T}_{n} \mathbf{Z}_{n}
$$

and

$$
\mathbf{E}_{n+1}=\mathbf{T}_{n} \mathbf{E}_{n} \mathbf{T}_{n}^{\dagger}
$$

Here we write

$$
\mathbf{E}_{n}=\left(\begin{array}{cc}
\mathbf{F}_{n} & \mathbf{C}_{n} \\
\mathbf{C}_{n}^{\dagger} & \mathbf{G}_{n}
\end{array}\right)
$$

where $\mathbf{F}_{n}, \mathbf{G}_{n}$ and $\mathbf{C}_{n}$ are two-by-two matrices. The projections of the beam ellipsoid onto the $X, X^{\prime}$ and $Y, Y^{\prime}$ planes are then the regions defined by [7]

$$
\left\{\mathbf{X}^{\dagger} \mathbf{F}_{n}^{-1} \mathbf{X}\right\} \leq \epsilon, \quad\left\{\mathbf{Y}^{\dagger} \mathbf{G}_{n}^{-1} \mathbf{Y}\right\} \leq \epsilon
$$

respectively. The areas of these regions are $\pi \epsilon_{x n}$ and $\pi \epsilon_{y n}$ where

$$
\epsilon_{x n}=\epsilon\left|\mathbf{F}_{n}\right|^{1 / 2}, \quad \epsilon_{y n}=\epsilon\left|\mathbf{G}_{n}\right|^{1 / 2}
$$

are the TBT horizontal and vertical emittances.

We shall assume that the initial beam ellipsoid has no coupling between the horizontal and vertical planes and that its projections onto these planes are matched to those of the matched ellipsoid. Since $\overline{\mathbf{w}}=\mathbf{w}^{\dagger}$ for the difference resonance, the two-by-two submatrices (78-80) of the matched ellipsoid matrix become

$$
\mathbf{F}=\mathcal{F} \mathcal{F}^{\dagger}, \quad \mathbf{G}=\mathcal{G G}^{\dagger}, \quad \mathbf{C}=\mathbf{0}
$$

where $\mathcal{F}$ and $\mathcal{G}$ are given by (176). Thus we have

$$
\mathbf{F}_{0}=a \mathcal{F F}^{\dagger}, \quad \mathbf{G}_{0}=b \mathcal{G} \mathcal{G}^{\dagger}, \quad \mathbf{C}_{0}=\mathbf{0}
$$

where $a$ and $b$ are positive constants (so that $\mathbf{E}_{0}$ is positive definite) and $a b=1$ (so that $\left|\mathbf{E}_{0}\right|=1$ ). The initial horizontal and vertical emittances are then

$$
\epsilon_{x 0}=a \epsilon, \quad \epsilon_{y 0}=b \epsilon
$$

and the TBT evolution of the emittances (194) is given by iteration of (191) with $\mathbf{T}_{n}$ given by (177-181) and with $\mathbf{E}_{0}$ given by (192) and (196). 


\subsection{Normalized TBT Equations}

Since the matrix $\mathcal{N}$ has been taken to be independent of $n$ it is convenient to introduce normalized matrices

$$
\mathcal{T}_{n}=\mathcal{N}^{-1} \mathbf{T}_{n} \mathcal{N}=\widehat{\mathbf{R}}_{n} \mathcal{U}_{n} \widehat{\mathbf{R}}_{n}^{-1}
$$

and

$$
\mathcal{E}_{n}=\mathcal{N}^{-1} \mathbf{E}_{n}\left(\mathcal{N}^{-1}\right)^{\dagger}
$$

We then have

$$
\mathcal{E}_{0}=\left(\begin{array}{cc}
a \mathbf{I} & \mathbf{0} \\
\mathbf{0} & b \mathbf{I}
\end{array}\right)
$$

and the recursion relation (191) becomes

$$
\mathcal{E}_{n+1}=\mathcal{T}_{n} \mathcal{E}_{n} \mathcal{T}_{n}^{\dagger}
$$

where

$$
\mathcal{T}_{n}=\left(\begin{array}{ll}
\mathbf{P}_{n} & \mathbf{q}_{n} \\
\mathbf{p}_{n} & \mathbf{Q}_{n}
\end{array}\right)
$$

and

$$
\begin{gathered}
\mathbf{P}_{n}=\mathcal{F}^{-1} \mathbf{M}_{n} \mathcal{F}=d_{n}^{2} \mathcal{A}_{n}+e_{n}^{2} \mathcal{B}_{n} \\
\mathbf{q}_{n}=\mathcal{F}^{-1} \mathbf{n}_{n} \mathcal{G}=d_{n} e_{n}\left\{\Omega^{\dagger} \mathcal{B}_{n}-\mathcal{A}_{n} \Omega^{\dagger}\right\} \\
\mathbf{p}_{n}=\mathcal{G}^{-1} \mathbf{m}_{n} \mathcal{F}=d_{n} e_{n}\left\{\mathcal{B}_{n} \Omega-\Omega \mathcal{A}_{n}\right\} \\
\mathbf{Q}_{n}=\mathcal{G}^{-1} \mathbf{N}_{n} \mathcal{G}=d_{n}^{2} \mathcal{B}_{n}+e_{n}^{2} \mathcal{A}_{n} .
\end{gathered}
$$

Partitioning $\mathcal{E}_{n}$ into two-by-two matrices we have

$$
\mathcal{E}_{n}=\left(\begin{array}{cc}
\mathcal{F}_{n} & \mathcal{C}_{n} \\
\mathcal{C}_{n}^{\dagger} & \mathcal{G}_{n}
\end{array}\right)
$$

where

$$
\mathcal{F}_{n}=\mathcal{F}^{-1} \mathbf{F}_{n}\left(\mathcal{F}^{-1}\right)^{\dagger}, \quad \mathcal{G}_{n}=\mathcal{G}^{-1} \mathbf{G}_{n}\left(\mathcal{G}^{-1}\right)^{\dagger}
$$

and

$$
\mathcal{C}_{n}=\mathcal{F}^{-1} \mathbf{C}_{n}\left(\mathcal{G}^{-1}\right)^{\dagger}
$$

Defining

$$
a_{n}=\left|\mathcal{F}_{n}\right|^{1 / 2}, \quad b_{n}=\left|\mathcal{G}_{n}\right|^{1 / 2}
$$

we then have

$$
\epsilon_{x n}=\epsilon\left|\mathbf{F}_{n}\right|^{1 / 2}=\epsilon\left|\mathcal{F}_{n}\right|^{1 / 2}=\epsilon a_{n}
$$


and

$$
\epsilon_{y n}=\epsilon\left|\mathbf{G}_{n}\right|^{1 / 2}=\epsilon\left|\mathcal{G}_{n}\right|^{1 / 2}=\epsilon b_{n}
$$

The recursion relation (201) along with the initial matrix $\mathcal{E}_{0}$ then gives the TBT emittances. Defining the product

$$
\mathcal{P}_{n}=\mathcal{T}_{n} \cdots \mathcal{T}_{1} \mathcal{T}_{0}
$$

we also have

$$
\mathcal{E}_{n+1}=\mathcal{P}_{n} \mathcal{E}_{0} \mathcal{P}_{n}^{\dagger}
$$

\subsection{Calculation of the TBT Emittances}

It follows from (203-206) that the two-by-two matrices $\mathbf{P}_{n}, \mathbf{q}_{n}, \mathbf{p}_{n}, \mathbf{Q}_{n}$ have the properties

$$
\begin{aligned}
& \overline{\mathbf{P}}_{n}=\mathbf{P}_{n}^{\dagger}, \quad \overline{\mathbf{q}}_{n}=\mathbf{q}_{n}^{\dagger} \\
& \overline{\mathbf{p}}_{n}=\mathbf{p}_{n}^{\dagger}, \quad \overline{\mathbf{Q}}_{n}=\mathbf{Q}_{n}^{\dagger}
\end{aligned}
$$

and are therefore of the form

$$
\mathbf{P}=\left(\begin{array}{rr}
P_{11} & P_{12} \\
-P_{12} & P_{11}
\end{array}\right)
$$

In Appendix $A$ it is shown that if $\mathcal{T}_{n}$ is a symplectic matrix with two-by-two submatrices that satisfy (215-216), and if $\mathcal{E}_{n}$ has the form

$$
\mathcal{E}_{n}=\left(\begin{array}{cc}
a_{n} \mathbf{I} & \mathcal{C}_{n} \\
\mathcal{C}_{n}^{\dagger} & b_{n} \mathbf{I}
\end{array}\right)
$$

where

$$
\overline{\mathcal{C}}_{n}=\mathcal{C}_{n}^{\dagger}
$$

then $\mathcal{E}_{n+1}$ will be of the form

$$
\mathcal{E}_{n+1}=\mathcal{T}_{n} \mathcal{E}_{n} \mathcal{T}_{n}^{\dagger}=\left(\begin{array}{cc}
a_{n+1} \mathbf{I} & \mathcal{C}_{n+1} \\
\mathcal{C}_{n+1}^{\dagger} & b_{n+1} \mathbf{I}
\end{array}\right)
$$

where

$$
\overline{\mathcal{C}}_{n+1}=\mathcal{C}_{n+1}^{\dagger}
$$

and

$$
a_{n+1}+b_{n+1}=a_{n}+b_{n}
$$


Since

$$
\mathcal{E}_{0}=\left(\begin{array}{cc}
a \mathbf{I} & \mathbf{0} \\
0 & b \mathbf{I}
\end{array}\right)
$$

it follows by induction that all of the matrices $\mathcal{E}_{n}$ are of the form (218) with

$$
\mathcal{C}_{n}=\left(\begin{array}{rr}
c_{n} & h_{n} \\
-h_{n} & c_{n}
\end{array}\right)
$$

satisfying (221) and with

$$
a_{n}+b_{n}=a_{0}+b_{0}=a+b .
$$

Thus the TBT sum of the horizontal and vertical emittances

$$
\epsilon_{x n}+\epsilon_{y n}=\epsilon\left\{a_{n}+b_{n}\right\}=\epsilon_{x 0}+\epsilon_{y 0}
$$

is conserved. This is a special case of the general theorem proved by Brown and Servranckx [7] which shows that one must have

$$
\epsilon_{x n}+\epsilon_{y n} \geq \epsilon_{x 0}+\epsilon_{y 0}
$$

Starting with the initial parameters $a_{0}=a, b_{0}=b, c_{0}=0$ and $h_{0}=0$, a computer program can be set up to calculate $a_{n}, b_{n}, c_{n}$ and $h_{n}$ recursively. This then gives the TBT emittances. The formulae for doing this are given in Appendix B.

\subsection{Approximate Expression for the TBT Emittances}

Using (198) in the product

$$
\mathcal{P}_{n}=\mathcal{T}_{n} \cdots \mathcal{T}_{1} \mathcal{T}_{0}
$$

we have

$$
\mathcal{P}_{n}=\left\{\widehat{\mathbf{R}}_{n} \mathcal{U}_{n} \widehat{\mathbf{R}}_{n}^{-1}\right\}\left\{\widehat{\mathbf{R}}_{n-1} \mathcal{U}_{n-1} \widehat{\mathbf{R}}_{n-1}^{-1}\right\} \cdots\left\{\widehat{\mathbf{R}}_{0} \mathcal{U}_{0} \widehat{\mathbf{R}}_{0}^{-1}\right\} .
$$

Here between each $U_{k+1}$ and $U_{k}$ we have the factor

$$
\widehat{\mathbf{R}}_{k+1}^{-1} \widehat{\mathbf{R}}_{k}=\left(\begin{array}{cc}
d_{k+1} \mathbf{I} & -e_{k+1} \Omega^{\dagger} \\
e_{k+1} \Omega & d_{k+1} \mathbf{I}
\end{array}\right)\left(\begin{array}{cc}
d_{k} \mathbf{I} & e_{k} \Omega^{\dagger} \\
-e_{k} \Omega & d_{k} \mathbf{I}
\end{array}\right)
$$


which we can write as

$$
\widehat{\mathbf{R}}_{k+1}^{-1} \widehat{\mathbf{R}}_{k}=\mathbf{I} \cos \left(\eta_{k+1}-\eta_{k}\right)+\mathbf{H} \sin \left(\eta_{k+1}-\eta_{k}\right)
$$

where

$$
\cos \eta_{j}=d_{j}, \quad \sin \eta_{j}=e_{j}
$$

and

$$
\mathbf{H}=\left(\begin{array}{cc}
\mathbf{0} & -\Omega^{\dagger} \\
\Omega & 0
\end{array}\right)
$$

Thus

$$
\widehat{\mathbf{R}}_{k+1}^{-1} \widehat{\mathbf{R}}_{k}=\mathbf{I}+\left(\eta_{k+1}-\eta_{k}\right) \mathbf{H}+\cdots
$$

and if $\eta_{k+1}-\eta_{k}$ is sufficiently small we may take

$$
\widehat{\mathbf{R}}_{k+1}^{-1} \widehat{\mathbf{R}}_{k}=\mathbf{I}
$$

This gives the approximate expression

$$
\mathcal{P}_{n}=\widehat{\mathbf{R}}_{n} \mathcal{V}_{n} \widehat{\mathbf{R}}_{0}^{-1}
$$

where

$$
\begin{gathered}
\mathcal{V}_{n}=\mathcal{U}_{n} \cdots \mathcal{U}_{1} \mathcal{U}_{0}=\left(\begin{array}{cc}
\mathbf{K}_{n} & \mathbf{0} \\
\mathbf{0} & \mathbf{L}_{n}
\end{array}\right) \\
\mathbf{K}_{n}=\mathcal{A}_{n} \cdots \mathcal{A}_{1} \mathcal{A}_{0}=\left(\begin{array}{cc}
\cos \Psi_{1 n} & \sin \Psi_{1 n} \\
-\sin \Psi_{1 n} & \cos \Psi_{1 n}
\end{array}\right) \\
\mathbf{L}_{n}=\mathcal{B}_{n} \cdots \mathcal{B}_{1} \mathcal{B}_{0}=\left(\begin{array}{rr}
\cos \Psi_{2 n} & \sin \Psi_{2 n} \\
-\sin \Psi_{2 n} & \cos \Psi_{2 n}
\end{array}\right) \\
\Psi_{1 n}=\psi_{1 n}+\psi_{1(n-1)}+\cdots+\psi_{1(1)}+\psi_{1(0)}
\end{gathered}
$$

and

$$
\Psi_{2 n}=\psi_{2 n}+\psi_{2(n-1)}+\cdots+\psi_{2(1)}+\psi_{2(0)} .
$$

Using

$$
\widehat{\mathbf{R}}_{n}=\left(\begin{array}{cc}
d_{n} \mathbf{I} & e_{n} \Omega^{\dagger} \\
-e_{n} \Omega & d_{n} \mathbf{I}
\end{array}\right), \quad \widehat{\mathbf{R}}_{0}^{-1}=\left(\begin{array}{cc}
d_{0} \mathbf{I} & -e_{0} \Omega^{\dagger} \\
e_{0} \Omega & d_{0} \mathbf{I}
\end{array}\right)
$$

we then have

$$
\mathcal{P}_{n}=\left(\begin{array}{cc}
\mathbf{P}_{n} & \mathbf{q}_{n} \\
\mathbf{p}_{n} & \mathbf{Q}_{n}
\end{array}\right)
$$


where

$$
\begin{gathered}
\mathbf{P}_{n}=d_{0} d_{n} \mathbf{K}_{n}+e_{0} e_{n} \mathbf{L}_{n} \\
\mathbf{q}_{n}=d_{0} e_{n} \Omega^{\dagger} \mathbf{L}_{n}-e_{0} d_{n} \mathbf{K}_{n} \Omega^{\dagger} \\
\mathbf{p}_{n}=e_{0} d_{n} \mathbf{L}_{n} \Omega-d_{0} e_{n} \Omega \mathbf{K}_{n} \\
\mathbf{Q}_{n}=d_{0} d_{n} \mathbf{L}_{n}+e_{0} e_{n} \mathbf{K}_{n} .
\end{gathered}
$$

Here we see that the two-by-two submatrices again satisfy the conditions

$$
\begin{aligned}
& \overline{\mathbf{P}}_{n}=\mathbf{P}_{n}^{\dagger}, \quad \overline{\mathbf{q}}_{n}=\mathbf{q}_{n}^{\dagger} \\
& \overline{\mathbf{p}}_{n}=\mathbf{p}_{n}^{\dagger}, \quad \overline{\mathbf{Q}}_{n}=\mathbf{Q}_{n}^{\dagger}
\end{aligned}
$$

and we therefore have

$$
\mathcal{E}_{n+1}=\mathcal{P}_{n} \mathcal{E}_{0} \mathcal{P}_{n}^{\dagger}=\left(\begin{array}{cc}
a_{n+1} \mathbf{I} & \mathcal{C}_{n+1} \\
\mathcal{C}_{n+1}^{\dagger} & b_{n+1} \mathbf{I}
\end{array}\right)
$$

where

$$
\overline{\mathcal{C}}_{n+1}=\mathcal{C}_{n+1}^{\dagger}
$$

and

$$
a_{n+1}+b_{n+1}=a+b .
$$

In Appendix $\mathrm{C}$ it is shown that

$$
\begin{aligned}
& a_{n+1}=F(n)-2(b-a) d_{0} e_{0} d_{n} e_{n} \cos \left(\Psi_{1 n}-\Psi_{2 n}\right) \\
& b_{n+1}=G(n)+2(b-a) d_{0} e_{0} d_{n} e_{n} \cos \left(\Psi_{1 n}-\Psi_{2 n}\right)
\end{aligned}
$$

where

$$
F(n)=a+(b-a)\left\{d_{0}^{2} e_{n}^{2}+e_{0}^{2} d_{n}^{2}\right\}
$$

and

$$
G(n)=b-(b-a)\left\{d_{0}^{2} e_{n}^{2}+e_{0}^{2} d_{n}^{2}\right\} .
$$

Although approximate, these equations are non-recursive and give the horizontal and vertical TBT emittances $\left(\epsilon_{x n}=\epsilon a_{n}, \epsilon_{y n}=\epsilon b_{n}\right)$ in terms of the known parameters $a, b, d_{0}, e_{0}, d_{n}, e_{n}, \Psi_{1 n}$ and $\Psi_{2 n}$.

Note that for the case in which the matrices $\mathcal{T}_{k}$ are all equal to $\mathcal{T}_{0}$ we have

$$
\mathcal{P}_{n}=\mathcal{T}_{0}^{n+1}=\widehat{\mathbf{R}}_{0} \mathcal{U}_{0}^{n+1} \widehat{\mathbf{R}}_{0}^{-1} .
$$

One then has

$$
a_{n+1}=a+2(b-a) d_{0}^{2} e_{0}^{2}\left\{1-\cos \Psi_{n}\right\}
$$


and

$$
b_{n+1}=b-2(b-a) d_{0}^{2} e_{0}^{2}\left\{1-\cos \Psi_{n}\right\}
$$

and in this case the equations are exact. Here

$$
\Psi_{n}=(n+1)\left(\psi_{10}-\psi_{20}\right)
$$

and

$$
d_{0}^{2} e_{0}^{2}=d_{0}^{2}\left(1-d_{0}^{2}\right)=\frac{K_{0}^{2}}{T_{0}^{2}+4 K_{0}^{2}}
$$

as shown in Section 3.6. Thus in this case the horizontal and vertical emittances oscillate with frequency $Q_{10}-Q_{20}$ and their sum remains constant. These equations are in agreement with equations (1) and (2) of Ref. [12].

\subsection{Comparison with Other Formulae}

For comparison of the approximate formulae (253-256) with those of Ref. [12] we note that

$$
\begin{aligned}
& d_{j}^{2}=\frac{1}{2}+\frac{\left|T_{j}\right|}{2\left|U_{j}\right|}=\frac{1}{2}\left\{\frac{U_{j}^{2}-T_{j}^{2}}{U_{j}^{2}-\left|T_{j} U_{j}\right|}\right\} \\
& e_{j}^{2}=\frac{1}{2}-\frac{\left|T_{j}\right|}{2\left|U_{j}\right|}=\frac{1}{2}\left\{\frac{U_{j}^{2}-T_{j}^{2}}{U_{j}^{2}+\left|T_{j} U_{j}\right|}\right\}
\end{aligned}
$$

and

which give

$$
U_{j}= \pm \sqrt{T_{j}^{2}+4 K_{j}^{2}}
$$

$$
d_{n}^{2}=\frac{1}{2}\left\{\frac{4 K_{n}^{2}}{T_{n}^{2}+4 K_{n}^{2}-\left|T_{n}\right| \sqrt{T_{n}^{2}+4 K_{n}^{2}}}\right\}
$$

and

$$
e_{n}^{2}=\frac{1}{2}\left\{\frac{4 K_{n}^{2}}{T_{n}^{2}+4 K_{n}^{2}+\left|T_{n}\right| \sqrt{T_{n}^{2}+4 K_{n}^{2}}}\right\} .
$$

For the case in which the initial matrix $\mathcal{T}_{0}$ is far from resonance we have

$$
d_{0}^{2} \approx 1, \quad e_{0}^{2} \approx 0
$$

and equations (255) and (256) become

$$
F(n)=a+\frac{1}{2}(b-a)\left\{\frac{4 K_{n}^{2}}{T_{n}^{2}+4 K_{n}^{2}+\left|T_{n}\right| \sqrt{T_{n}^{2}+4 K_{n}^{2}}}\right\}
$$


and

$$
G(n)=b-\frac{1}{2}(b-a)\left\{\frac{4 K_{n}^{2}}{T_{n}^{2}+4 K_{n}^{2}+\left|T_{n}\right| \sqrt{T_{n}^{2}+4 K_{n}^{2}}}\right\} .
$$

These equations are of the same form as equations (5) and (6) of Ref. [12].

\subsection{TBT Emittance Examples}

For $0 \leq n \leq N$ let the TBT tunes be given by

$$
Q_{1 n}=Q+\frac{1}{2} \Delta Q_{n}, \quad Q_{2 n}=Q-\frac{1}{2} \Delta Q_{n}
$$

where

$$
\Delta Q_{n}=\sqrt{\Delta_{n}^{2}+\left(\Delta Q_{\min }\right)^{2}}
$$

and

$$
\Delta_{n}^{2}=\left(\frac{n-N}{N}\right)^{2}\left\{\left(\Delta Q_{\max }\right)^{2}-\left(\Delta Q_{\min }\right)^{2}\right\}
$$

The average of $Q_{1 n}$ and $Q_{2 n}$ is then constant while the difference $Q_{1 n}-Q_{2 n}$ varies from $\Delta Q_{\max }$ at $n=0$ to $\Delta Q_{\min }$ at $n=N$. We assume here that $Q_{1 n}, Q_{2 n}$ and $Q$ are all between 0 and 0.5 .

For $N+1 \leq N+k \leq 2 N$ we let

$$
\Delta Q_{N+k}=\Delta Q_{N-k}
$$

which gives

$$
Q_{1(N+k)}=Q_{1(N-k)}, \quad Q_{2(N+k)}=Q_{2(N-k)} .
$$

Having specified the values of $Q_{1 n}$ and $Q_{2 n}$ we can calculate

$$
U_{n}=2 \cos \left(2 \pi Q_{1 n}\right)-2 \cos \left(2 \pi Q_{2 n}\right)
$$

and

$$
T_{n}^{2}=U_{n}^{2}-4 K_{n}^{2}
$$

Here we shall assume that $K_{n}$ is independent of $n$ with

$$
4 K_{n}^{2}=U_{N}^{2}
$$

for all $n$. This gives

$$
T_{n}^{2}=U_{n}^{2}-U_{N}^{2}
$$


and

$$
T_{N}^{2}=0
$$

We then have

$$
d_{n}^{2}=\frac{1}{2}+\frac{T_{n}}{2 U_{n}}
$$

and

$$
e_{n}^{2}=\frac{1}{2}-\frac{T_{n}}{2 U_{n}}
$$

where $T_{n}$ is taken to have the same sign as $U_{n}$ and $d_{n}$ is taken to be positive. We also take $e_{n} \geq 0$ for $T_{n} \leq 0$ and $e_{n} \leq 0$ for $T_{n}>0$ as discussed in Section 4.1. It then follows from (273-281) that

$$
U_{N+k}=U_{N-k}, \quad T_{N+k}=T_{N-k}
$$

and

$$
d_{N+k}=d_{N-k}, \quad e_{N+k}=e_{N-k} .
$$

Taking $2 N=4000$ turns, $Q=0.25, \Delta Q_{\max }=0.05$ and $\Delta Q_{\min }=0.005$ we obtain the values of $Q_{1 n}$ and $Q_{2 n}$ plotted in Figure 4. The corresponding values of $T_{n}, U_{n}, d_{n}$ and $e_{n}$ are plotted in Figure 5. Here we see that $T_{n}$ increases monotonically until it reaches reaches zero at $n=N=2000$. Rather than passing through zero it then decreases monotonically until it reaches the value $T_{0}$ at $n=2 N=4000$. Note that since $T_{n} \leq 0$ for all $n$, we have $e_{n} \geq 0$ for all $n$.

The exact TBT horizontal and vertical emittances $\epsilon_{x n}$ and $\epsilon_{y n}$ obtained under these conditions (and with parameter $\omega=\pi / 4$ ) are plotted in Figure 6. Here the emittances were obtained by iteration of equation (201) with starting parameters $\epsilon_{x 0}=0.7, \epsilon_{y 0}=0.3, c_{0}=0$ and $h_{0}=0$. Note that the emittances are not exchanged in this case. In the next section we will see that exchange becomes possible if $T_{n}$ passes through zero. The corresponding elements $c_{n}$ and $h_{n}$ of normalized beam ellipsoid matrix $\mathcal{E}_{n}$ are plotted in Figure 7.

The approximate horizontal and vertical emittances obtained from equations (253-256) are plotted in Figure 8. These are overlayed on the exact emittances in Figure 9. Here we see that there is good agreement up to turn 2000. This is also seen in Figure $\mathbf{1 0}$ where the differences (approximate minus exact) are plotted. Note that for both the exact and approximate emittances the sum of the horizontal and vertical emittances is rigorously conserved as shown in Sections 4.4 and 4.5. 
The condition for good agreement between the exact and approximate emittances is that the parameter $\eta_{n}$ defined by equations (232) vary sufficiently slowly. A possible criterion for slow variation is suggested by the condition for adiabatic invariance [13]. One may expect that if the change in $\eta_{n}$ over one period of oscillation at the difference frequency $Q_{1}-Q_{2}$ is small compared to $\eta_{n}$, then the difference between the approximate and exact emittances will remain small. Let us define adiabatic parameter

$$
\xi_{n}=\tau_{n}\left\{\frac{\eta_{n+1}-\eta_{n}}{\left|\eta_{n}\right|}\right\}
$$

where

$$
\tau_{n}=\left|1 / \Delta Q_{n}\right|
$$

is the period of oscillation at the difference frequency. The criterion for sufficiently slow variation of $\eta_{n}$ then becomes

$$
\xi_{n} \ll 1 \text {. }
$$

The parameter $\xi_{n}$ is plotted in Figure 5. Here we see that the condition (286) is satisfied except in the region near $n=2000$. The agreement between exact and approximate emittances is actually good up to $n=2000$ but then becomes poor on subsequent turns. In order to improve the agreement we can reduce $\eta_{n+1}-\eta_{n}$ by reducing $\Delta Q_{\max }$ from 0.05 to 0.025 . We then obtain the parameters $T_{n}, U_{n}, d_{n}, e_{n}$ and $\xi_{n}$ plotted in Figure 11. Here we see that $\xi_{n}$ is reduced by a factor of 2 . The corresponding exact and approximate emittances and their differences are plotted in Figures 12, 13 and 14. Comparing with Figures 9 and 10 we see that the difference between exact and approximate emittances is also reduced by a factor of 2 .

\subsection{Approximate Equations for Emittance Exchange}

In the previous section we found that the horizontal and vertical emittances are not exchanged even though $T_{n}$ goes to zero. Here we show that exchange can occur if $T_{n}$ passes through zero.

For $0 \leq n \leq N$ we let the TBT tunes be given by (270-272) as before, but now for

$$
N+1 \leq N+k \leq 2 N
$$

we take

$$
Q_{1(N+k)}=Q_{2(N-k)}, \quad Q_{2(N+k)}=Q_{1(N-k)} .
$$


Thus the tunes $Q_{1 n}$ and $Q_{2 n}$ are "flipped" for $n>N$ and we have

$$
U_{N+k}=2 \cos \left\{2 \pi Q_{2(N-k)}\right\}-2 \cos \left\{2 \pi Q_{1(N-k)}\right\}=-U_{N-k} .
$$

It then follows that

$$
T_{N+k}=-T_{N-k}
$$

and

$$
d_{N+k}=d_{N-k}, \quad e_{N+k}=-e_{N-k} .
$$

(Here as before we take $e_{n} \geq 0$ for $T_{n} \leq 0$ and $e_{n} \leq 0$ for $T_{n}>0$.)

For $m=N+k$ we have

$$
\mathcal{E}_{m+1}=\left(\begin{array}{cc}
a_{m+1} \mathbf{I} & \mathcal{C}_{m+1} \\
\mathcal{C}_{m+1}^{\dagger} & b_{m+1} \mathbf{I}
\end{array}\right)=\mathcal{Q}_{k} \mathcal{P}_{N} \mathcal{E}_{0} \mathcal{P}_{N}^{\dagger} \mathcal{Q}_{k}^{\dagger}
$$

where

$$
\mathcal{Q}_{k}=\mathcal{T}_{N+k} \cdots \mathcal{T}_{N+2} \mathcal{T}_{N+1}
$$

and

$$
\mathcal{P}_{N}=\mathcal{T}_{N} \cdots \mathcal{T}_{1} \mathcal{T}_{0}
$$

Here

$$
\begin{gathered}
\mathcal{T}_{N+k}=\widehat{\mathbf{R}}_{N+k} \mathcal{U}_{N+k} \widehat{\mathbf{R}}_{N+k}^{-1} \\
\widehat{\mathbf{R}}_{N+k}=\left(\begin{array}{cc}
d_{N-k} \mathbf{I} & -e_{N-k} \Omega^{\dagger} \\
e_{N-k} \Omega & d_{N-k} \mathbf{I}
\end{array}\right)=\widehat{\mathbf{R}}_{N-k}^{-1}
\end{gathered}
$$

and

$$
\mathcal{U}_{N+k}=\left(\begin{array}{cc}
\mathcal{B}_{N-k} & \mathbf{0} \\
\mathbf{0} & \mathcal{A}_{N-k}
\end{array}\right)=\mathbf{J} \mathcal{U}_{N-k} \mathbf{J}^{-1}
$$

where

$$
\mathbf{J}=\left(\begin{array}{cc}
\mathbf{0} & \mathbf{I} \\
\mathbf{I} & \mathbf{0}
\end{array}\right), \quad \mathbf{J}^{-1}=\mathbf{J} .
$$

We then have the approximate equations

$$
\mathcal{Q}_{k}=\widehat{\mathbf{R}}_{N-k}^{-1}\left\{\mathbf{J} \mathcal{U}_{N-k} \mathbf{J}\right\} \cdots\left\{\mathbf{J} \mathcal{U}_{N-1} \mathbf{J}\right\} \widehat{\mathbf{R}}_{N-1}
$$

and

$$
\mathcal{Q}_{k} \mathcal{P}_{N}=\left\{\widehat{\mathbf{R}}_{N-k}^{-1} \mathbf{J} \mathcal{M}_{k} \mathbf{J} \widehat{\mathbf{R}}_{N-1}\right\}\left\{\widehat{\mathbf{R}}_{N} \mathcal{V}_{N} \widehat{\mathbf{R}}_{0}^{-1}\right\}
$$


where

$$
\begin{gathered}
\mathcal{M}_{k}=\mathcal{U}_{N-1} \cdots \mathcal{U}_{N-k}=\left(\begin{array}{cc}
\mathcal{K}_{k} & 0 \\
0 & \mathcal{L}_{k}
\end{array}\right) \\
\mathcal{K}_{k}=\mathcal{A}_{N-1} \cdots \mathcal{A}_{N-k}=\left(\begin{array}{rr}
\cos \Phi_{1 k} & \sin \Phi_{1 k} \\
-\sin \Phi_{1 k} & \cos \Phi_{1 k}
\end{array}\right) \\
\mathcal{L}_{k}=\mathcal{B}_{N-1} \cdots \mathcal{B}_{N-k}=\left(\begin{array}{rr}
\cos \Phi_{2 k} & \sin \Phi_{2 k} \\
-\sin \Phi_{2 k} & \cos \Phi_{2 k}
\end{array}\right) \\
\Phi_{1 k}=\psi_{1(N-1)}+\cdots+\psi_{1(N-k)} \\
\Phi_{2 k}=\psi_{2(N-1)}+\cdots+\psi_{2(N-k)}
\end{gathered}
$$

and $\mathcal{V}_{N}$ is given by $(237-241)$ with $n=N$.

Note that because $e_{n}$ changes sign as $T_{n}$ passes through zero, the approximation

$$
\widehat{\mathbf{R}}_{j+1}^{-1} \widehat{\mathbf{R}}_{j}=\mathbf{I}
$$

is not valid for $j=N$. Instead, using (296) we have

$$
\widehat{\mathbf{R}}_{N+1}^{-1} \widehat{\mathbf{R}}_{N}=\widehat{\mathbf{R}}_{N-1} \widehat{\mathbf{R}}_{N} .
$$

Thus using the valid approximation

$$
\widehat{\mathbf{R}}_{N}^{-1} \widehat{\mathbf{R}}_{N-1}=\mathbf{I}
$$

we have

$$
\begin{gathered}
\widehat{\mathbf{R}}_{N-1}=\widehat{\mathbf{R}}_{N} \widehat{\mathbf{R}}_{N}^{-1} \widehat{\mathbf{R}}_{N-1}=\widehat{\mathbf{R}}_{N} \\
\widehat{\mathbf{R}}_{N+1}^{-1} \widehat{\mathbf{R}}_{N}=\widehat{\mathbf{R}}_{N-1} \widehat{\mathbf{R}}_{N}=\widehat{\mathbf{R}}_{N}^{2}
\end{gathered}
$$

and

$$
\mathcal{Q}_{k} \mathcal{P}_{N}=\widehat{\mathbf{R}}_{N-k}^{-1}\left\{\mathbf{J} \mathcal{M}_{k} \mathbf{J}\right\} \widehat{\mathbf{R}}_{N}^{2} \mathcal{V}_{N} \widehat{\mathbf{R}}_{0}^{-1}
$$

where

$$
\widehat{\mathbf{R}}_{N}^{2}=\left(\begin{array}{cc}
\left(2 d_{N}^{2}-1\right) \mathbf{I} & 2 d_{N} e_{N} \Omega^{\dagger} \\
-2 d_{N} e_{N} \Omega & \left(2 d_{N}^{2}-1\right) \mathbf{I}
\end{array}\right)
$$

Here

$$
d_{N}=e_{N}=\frac{1}{\sqrt{2}}
$$

which gives

$$
\widehat{\mathbf{R}}_{N}^{2}=\left(\begin{array}{cc}
0 & \Omega^{\dagger} \\
-\Omega & 0
\end{array}\right)
$$


and

$$
\left\{\mathbf{J} \mathcal{M}_{k} \mathbf{J}\right\} \widehat{\mathbf{R}}_{N}^{2} \mathcal{V}_{N}=\left(\begin{array}{cc}
0 & \mathbf{L} \Omega^{\dagger} \\
-\mathbf{K} \Omega & 0
\end{array}\right)
$$

where

$$
\mathbf{K}=\mathcal{K}_{k} \mathbf{K}_{N}, \quad \mathbf{L}=\mathcal{L}_{k} \mathbf{L}_{N}
$$

Using

$$
\mathbf{R}_{0}^{-1}=\left(\begin{array}{cc}
d_{0} \mathbf{I} & -e_{0} \Omega^{\dagger} \\
e_{0} \Omega & d_{0} \mathbf{I}
\end{array}\right)
$$

and

$$
\mathbf{R}_{N-k}^{-1}=\left(\begin{array}{cc}
d_{N-k} \mathbf{I} & -e_{N-k} \Omega^{\dagger} \\
e_{N-k} \Omega & d_{N-k} \mathbf{I}
\end{array}\right)
$$

we then have

$$
\begin{gathered}
\left\{\mathbf{J} \mathcal{M}_{k} \mathbf{J}\right\} \widehat{\mathbf{R}}_{N}^{2} \mathcal{V}_{N} \mathbf{R}_{0}^{-1}=\left(\begin{array}{cc}
\mathbf{0} & \mathbf{L} \Omega^{\dagger} \\
-\mathbf{K} \Omega & \mathbf{0}
\end{array}\right)\left(\begin{array}{cc}
d_{0} \mathbf{I} & -e_{0} \Omega^{\dagger} \\
e_{0} \Omega & d_{0} \mathbf{I}
\end{array}\right) \\
\left\{\mathbf{J} \mathcal{M}_{k} \mathbf{J}\right\} \widehat{\mathbf{R}}_{N}^{2} \mathcal{V}_{N} \mathbf{R}_{0}^{-1}=\left(\begin{array}{cc}
e_{0} \mathbf{L} & d_{0} \mathbf{L} \Omega^{\dagger} \\
-d_{0} \mathbf{K} \Omega & e_{0} \mathbf{K}
\end{array}\right)
\end{gathered}
$$

and

$$
\mathcal{Q}_{k} \mathcal{P}_{N}=\mathbf{R}_{N-k}^{-1}\left\{\mathbf{J} \mathcal{M}_{k} \mathbf{J}\right\} \widehat{\mathbf{R}}_{N}^{2} \mathcal{V}_{N} \mathbf{R}_{0}^{-1}=\left(\begin{array}{cc}
\mathbf{P} & \mathbf{q} \\
\mathbf{p} & \mathbf{Q}
\end{array}\right)
$$

where

$$
\left(\begin{array}{ll}
\mathbf{P} & \mathbf{q} \\
\mathbf{p} & \mathbf{Q}
\end{array}\right)=\left(\begin{array}{cc}
d_{N-k} \mathbf{I} & -e_{N-k} \Omega^{\dagger} \\
e_{N-k} \Omega & d_{N-k} \mathbf{I}
\end{array}\right)\left(\begin{array}{cc}
e_{0} \mathbf{L} & d_{0} \mathbf{L} \Omega^{\dagger} \\
-d_{0} \mathbf{K} \Omega & e_{0} \mathbf{K}
\end{array}\right)
$$

Thus

$$
\begin{gathered}
\mathbf{P}=e_{0} d_{N-k} \mathbf{L}+d_{0} e_{N-k} \mathbf{K} \\
\mathbf{q}=d_{0} d_{N-k} \mathbf{L} \Omega^{\dagger}-e_{0} e_{N-k} \Omega^{\dagger} \mathbf{K} \\
\mathbf{p}=e_{0} e_{N-k} \Omega \mathbf{L}-d_{0} d_{N-k} \mathbf{K} \Omega \\
\mathbf{Q}=d_{0} e_{N-k} \mathbf{L}+e_{0} d_{N-k} \mathbf{K}
\end{gathered}
$$

and we see that again we have

$$
\begin{aligned}
& \overline{\mathbf{P}}=\mathbf{P}^{\dagger}, \quad \overline{\mathbf{q}}=\mathbf{q}^{\dagger} \\
& \overline{\mathbf{p}}=\mathbf{p}^{\dagger}, \quad \overline{\mathbf{Q}}=\mathbf{Q}^{\dagger}
\end{aligned}
$$


As shown in Appendix $\mathrm{C}$ we then have

$$
\begin{aligned}
& a_{m+1}=G(N-k)-2(b-a) d_{0} e_{0} d_{N-k} e_{N-k} \cos \left(\theta_{1}-\theta_{2}\right) \\
& b_{m+1}=F(N-k)+2(b-a) d_{0} e_{0} d_{N-k} e_{N-k} \cos \left(\theta_{1}-\theta_{2}\right)
\end{aligned}
$$

where $m=N+k$,

$$
\begin{aligned}
& \theta_{1}=\psi_{1(N-1)}+\psi_{1(N-2)}+\cdots+\psi_{1(N-k)}+\Psi_{1 N} \\
& \theta_{2}=\psi_{2(N-1)}+\psi_{2(N-2)}+\cdots+\psi_{2(N-k)}+\Psi_{2 N}
\end{aligned}
$$

and

$$
\begin{aligned}
& F(n)=a+(b-a)\left\{d_{0}^{2} e_{n}^{2}+e_{0}^{2} d_{n}^{2}\right\} \\
& G(n)=b-(b-a)\left\{d_{0}^{2} e_{n}^{2}+e_{0}^{2} d_{n}^{2}\right\}
\end{aligned}
$$

(The phases $\Psi_{1 N}$ and $\Psi_{2 N}$ are given by (240-241) with $n=N$.) Here again we have equations that, although approximate, are non-recursive and give the horizontal and vertical TBT emittances in terms of the known parameters $a, b, d_{0}, e_{0}, d_{n}, e_{n}, \theta_{1}$ and $\theta_{2}$.

Note that since

$$
d_{N+k}=d_{N-k}, \quad e_{N+k}=-e_{N-k}
$$

we have

$$
\begin{aligned}
& G(N-k)=G(N+k)=G(m) \\
& F(N-k)=F(N+k)=F(m)
\end{aligned}
$$

and equations (329-330) can be written as

$$
\begin{aligned}
& a_{m+1}=G(m)+2(b-a) d_{0} e_{0} d_{m} e_{m} \cos \left(\theta_{1}-\theta_{2}\right) \\
& b_{m+1}=F(m)-2(b-a) d_{0} e_{0} d_{m} e_{m} \cos \left(\theta_{1}-\theta_{2}\right)
\end{aligned}
$$

where

$$
N+1 \leq m \leq 2 N
$$




\subsection{Demonstration of Emittance Exchange}

Now taking $m=N+k=2 N$ we have

$$
\begin{aligned}
& a_{2 N+1}=G(0)-2(b-a) d_{0}^{2} e_{0}^{2} \cos \left(\theta_{1}-\theta_{2}\right) \\
& b_{2 N+1}=F(0)+2(b-a) d_{0}^{2} e_{0}^{2} \cos \left(\theta_{1}-\theta_{2}\right)
\end{aligned}
$$

where

$$
\theta_{1}=2 \Psi_{1 N}-\psi_{1 N}, \quad \theta_{2}=2 \Psi_{2 N}-\psi_{2 N}
$$

and

$$
\begin{aligned}
& G(0)=b-2(b-a) d_{0}^{2} e_{0}^{2} \\
& F(0)=a+2(b-a) d_{0}^{2} e_{0}^{2}
\end{aligned}
$$

Thus

$$
a_{2 N+1}=b-2(b-a) d_{0}^{2}\left(1-d_{0}^{2}\right)\left\{1+\cos \left(\theta_{1}-\theta_{2}\right)\right\}
$$

and

$$
b_{2 N+1}=a+2(b-a) d_{0}^{2}\left(1-d_{0}^{2}\right)\left\{1+\cos \left(\theta_{1}-\theta_{2}\right)\right\}
$$

where

$$
d_{0}^{2}\left(1-d_{0}^{2}\right)=\frac{K_{0}^{2}}{T_{0}^{2}+4 K_{0}^{2}}
$$

Here we see that if $K_{0}$ is small or if $T_{0}$ is large then $d_{0}^{2}\left(1-d_{0}^{2}\right) \approx 0$ and equations (346-347) become

$$
a_{2 N+1} \approx b, \quad b_{2 N+1} \approx a .
$$

Since $a_{0}=a$ and $b_{0}=b$, we see that the TBT emittances $\left(\epsilon_{x n}=\epsilon a_{n}\right.$, $\left.\epsilon_{y n}=\epsilon b_{n}\right)$ are exchanged after $2 N$ turns.

\subsection{Emittance Exchange Examples}

Taking $2 N=4000$ turns, $Q=0.25, \Delta Q_{\max }=0.05$ and $\Delta Q_{\min }=0.005$ in equations (270-272) and (287-288) we obtain the values of $Q_{1 n}$ and $Q_{2 n}$ plotted in Figure 15. Note that in this case the tunes "flip" at $n=N=2000$. The corresponding values of $T_{n}, U_{n}, d_{n}, e_{n}$ and $\xi_{n}$ are plotted in Figure 16. Here we see that $T_{n}$ is monotonically increasing and passes through zero at $n=N=2000$. For $n \leq N$ we have $T_{n} \leq 0$ and $e_{n}>0$, while for $n>N$ we have $T_{n}>0$ and $e_{n}<0$.

The exact TBT horizontal and vertical emittances $\epsilon_{x n}$ and $\epsilon_{y n}$ obtained under these conditions (and with parameter $\omega=\pi / 4$ ) are plotted in 
Figure 17. Here the emittances were obtained by iteration of equations (201) and (292) with starting parameters $\epsilon_{x 0}=0.7, \epsilon_{y 0}=0.3, c_{0}=0$ and $h_{0}=0$. Note that the emittances are indeed exchanged in this case. The corresponding elements $c_{n}$ and $h_{n}$ of the normalized beam ellipsoid matrix $\mathcal{E}_{n}$ are plotted in Figure 18.

The approximate horizontal and vertical emittances obtained from equations (253-254) and (338-339) are plotted in Figure 19. These are overlayed on the exact emittances in Figure 20. Here we see that there is good agreement over the whole range of 4000 turns. This is also seen in Figure 21 where the differences (approximate minus exact) are plotted. In this case there is good agreement even though the parameter $\xi_{n}$ plotted in Figure 16 does not satisfy the adiabatic condition (286) in the region near $n=2000$.

Let us now take $2 N=500$ turns but keep the other parameters $(Q$, $\Delta Q_{\max }, \Delta Q_{\min }, \epsilon_{x 0}, \epsilon_{y 0}, c_{0}, h_{0}$ and $\left.\omega\right)$ the same as in the previous example. The resonance is then traversed $4000 / 500=8$ times faster than before. This means that the adiabatic parameter $\xi_{n}$ will be 8 times larger. The exact TBT horizontal and vertical emittances $\epsilon_{x n}$ and $\epsilon_{y n}$ obtained under these conditions are plotted in Figure 22. Here we see that there is partial exchange of emittances with large oscillations after turn 250. In Figure 23 the approximate emittances are overlayed on top of the exact emittances. The differences (approximate minus exact) are plotted in Figure 24. As expected the agreement between the approximate and exact emittances is poor starting around turn 250 .

\section{Summary}

We have shown that the Edwards-Teng parameterization along with the normalized coupling matrix allows for classification of the one-turn matirx in terms of parameters $A$ and $B$. For $A \gg B$ the one-turn matrix gives TBT amplitudes in horizontal and vertical planes that are characteristic of the difference resonance while for $B \gg A$ the amplitudes are characteristic of the sum resonance. The conditions $B=0$ and $A=0$ give one-turn matrices of the same form as those obtained in the hamiltonian treatment of the difference and sum resonances respectively. Taking $B=0$ gives horizontal and vertical amplitudes that contain only the frequency $Q_{1}-Q_{2}$, while $A=0$ gives amplitudes that contain only the frequency $Q_{1}+Q_{2}$. This is equivalent to discarding the high-frequency terms in the 
hamiltonian treatment of the resonances. The connection between the hamiltonian and matrix treatments is discussed further in Ref. [14].

Having established that the condition $B=0$ is associated with the difference resonance, we use matrices that satisfy this condition to study the TBT evolution of a distribution of beam particles under the influence of the resonance. Rather than track individual particles we track the parameters of the beam ellipsoid containing the distribution. In particular we track the parameters of the beam ellipsoid projections onto the. horizontal and vertical planes; these give the horizontal and vertical emittances. Tracking the ellipsoid parameters amounts to iteration of the equation

$$
\mathbf{E}_{n+1}=\mathbf{T}_{n} \mathbf{E}_{n} \mathbf{T}_{n}^{\dagger}
$$

as described in the text. It is assumed that the Courant-Snyder parameters appearing in the matrices $\mathbf{T}_{n}$ are independent of $n$. This simplification leads to the normalized equation

$$
\mathcal{E}_{n+1}=\mathcal{T}_{n} \mathcal{E}_{n} \mathcal{T}_{n}^{\dagger}
$$

which paves the way for a set of approximate non-recursive equations giving the TBT emittances. These equations are of the same form as those of Ref. [12]. The approximate emittances agree well with the "exact" ones obtained iteratively provided the "adiabatic" condition described in the text is satisfied. For both the approximate and exact emittances, the TBT sum of the horizontal and vertical emittances is rigorously conserved. Both the exact and approximate equations demonstrate that the exchange of horizontal and vertical emittances can occur when the matrix parameter $T_{n}$ passes through zero.

\section{Appendix A}

Let

$$
\mathcal{T}=\left(\begin{array}{cc}
\mathbf{P} & \mathbf{q} \\
\mathbf{p} & \mathbf{Q}
\end{array}\right)
$$

be a four-by-four symplectic matrix where $\mathbf{P}, \mathbf{q}, \mathbf{p}$, and $\mathbf{Q}$ are two-by-two matrices that satisfy

$$
\begin{gathered}
\overline{\mathbf{P}}=\mathbf{P}^{\dagger}, \quad \overline{\mathbf{q}}=\mathbf{q}^{\dagger} \\
\overline{\mathbf{p}}=\mathbf{p}^{\dagger}, \quad \overline{\mathbf{Q}}=\mathbf{Q}^{\dagger}
\end{gathered}
$$


And let

$$
\mathcal{E}_{0}=\left(\begin{array}{cc}
a_{0} \mathbf{I} & \mathcal{C}_{0} \\
\mathcal{C}_{0}^{\dagger} & b_{0} \mathbf{I}
\end{array}\right)
$$

where $a_{0}$ and $b_{0}$ are numbers and $\mathcal{C}_{0}$ is a two-by-two matrix that satisfies

$$
\overline{\mathcal{C}}_{0}=\mathcal{C}_{0}^{\dagger} \text {. }
$$

Writing

$$
\mathcal{E}_{1}=\mathcal{T} \mathcal{E}_{0} \mathcal{T}^{\dagger}=\left(\begin{array}{cc}
\mathcal{F}_{1} & \mathcal{C}_{1} \\
\mathcal{C}_{1}^{\dagger} & \mathcal{G}_{1}
\end{array}\right)
$$

we want to show that

$$
\mathcal{F}_{1}=a_{1} \mathbf{I}, \quad \mathcal{G}_{1}=b_{1} \mathbf{I}
$$

and

$$
\overline{\mathcal{C}}_{1}=\mathcal{C}_{1}^{\dagger}
$$

where $a_{1}$ and $b_{1}$ are numbers and

$$
a_{1}+b_{1}=a_{0}+b_{0}
$$

Carrying out the matrix multiplications in (357) and using (353-354) we find that

$$
\begin{aligned}
& \mathcal{F}_{1}=a_{0} \mathbf{P} \overline{\mathbf{P}}+b_{0} \mathbf{q} \overline{\mathbf{q}}+\mathbf{q} \overline{\mathcal{C}}_{0} \overline{\mathbf{P}}+\mathbf{P} \mathcal{C}_{0} \overline{\mathbf{q}} \\
& \mathcal{G}_{1}=a_{0} \mathbf{p} \overline{\mathbf{p}}+b_{0} \mathbf{Q} \overline{\mathbf{Q}}+\mathbf{Q} \overline{\mathcal{C}}_{0} \overline{\mathbf{p}}+\mathbf{p} \mathcal{C}_{0} \overline{\mathbf{Q}}
\end{aligned}
$$

and

$$
\mathcal{C}_{1}=a_{0} \mathbf{P} \overline{\mathbf{p}}+b_{0} \mathbf{q} \overline{\mathbf{Q}}+\mathbf{q} \overline{\mathcal{C}}_{0} \overline{\mathbf{p}}+\mathbf{P} \mathcal{C}_{0} \overline{\mathbf{Q}}
$$

Then using symplectic conjugate properties (12) and (13) we have

$$
\mathcal{F}_{1}=a_{1} \mathbf{I}, \quad \mathcal{G}_{1}=b_{1} \mathbf{I}
$$

where

$$
a_{1}=a_{0}|\mathbf{P}|+b_{0}|\mathbf{q}|+\operatorname{Tr}\left(\mathbf{q} \overline{\mathcal{C}}_{0} \overline{\mathbf{P}}\right)
$$

and

$$
b_{1}=a_{0}|\mathbf{p}|+b_{0}|\mathbf{Q}|+\operatorname{Tr}\left(\mathbf{Q} \overline{\mathcal{C}}_{0} \overline{\mathbf{p}}\right) \text {. }
$$

This proves (358). Using (353-354) we also have

$$
\mathcal{C}_{1}^{\dagger}=a_{0} \mathbf{p} \overline{\mathbf{P}}+b_{0} \mathbf{Q} \overline{\mathbf{q}}+\mathbf{p} \mathcal{C}_{0} \overline{\mathbf{q}}+\mathbf{Q} \overline{\mathcal{C}}_{0} \overline{\mathbf{P}}=\overline{\mathcal{C}}_{1}
$$

which proves (359). 
Now since the matrix $\mathcal{T}$ is symplectic, its two-by-two submatrices must satisfy

$$
\begin{array}{cc}
|\mathbf{P}|+|\mathbf{p}|=1, & |\mathbf{Q}|+|\mathbf{q}|=1 \\
|\mathbf{P}|=|\mathbf{Q}|, & |\mathbf{p}|=|\mathbf{q}|
\end{array}
$$

and

$$
\overline{\mathbf{P}} \mathbf{q}+\overline{\mathbf{p}} \mathbf{Q}=\mathbf{0}, \quad \mathbf{P} \overline{\mathbf{p}}+\mathbf{q} \overline{\mathbf{Q}}=\mathbf{0}
$$

as shown by Brown and Servranckx [7]. We therefore have

$$
a_{1}+b_{1}=a_{0}+b_{0}+\operatorname{Tr}\left(\mathbf{q} \overline{\mathcal{C}}_{0} \overline{\mathbf{P}}\right)+\operatorname{Tr}\left(\mathbf{Q} \overline{\mathcal{C}}_{0} \overline{\mathbf{p}}\right)
$$

and

$$
\operatorname{Tr}\left(\mathbf{q} \overline{\mathcal{C}}_{0} \overline{\mathbf{P}}\right)+\operatorname{Tr}\left(\mathbf{Q} \overline{\mathcal{C}}_{0} \overline{\mathbf{p}}\right)=\operatorname{Tr}\left\{\overline{\mathcal{C}}_{0}(\overline{\mathbf{P}} \mathbf{q}+\overline{\mathbf{p}} \mathbf{Q})\right\}=0
$$

which proves (360).

The sum of the projected horizontal and vertical emittances is therefore conserved under the assumed conditions. Moreover, if another transfer matrix $\mathcal{T}_{1}$ having the same properties as $\mathcal{T}$ is applied to form $\mathcal{E}_{2}=\mathcal{T}_{1} \mathcal{E}_{1} \mathcal{T}_{1}^{\dagger}$, the sum of the emittances will again be conserved. Thus, if we proceed in a series of steps with transfer matrices $\mathcal{I}_{n}$ all having the same properties as $\mathcal{T}$, the sum of the projected horizontal and vertical emittances will remain constant.

\section{Appendix B}

Here we work out the details of the computation of $a_{1}, b_{1}$ and $\mathcal{C}_{1}$.

Equations (368) and (369) give

$$
|\mathbf{q}|=1-|\mathbf{P}|
$$

so the expression for $a_{1}$ becomes

$$
\begin{aligned}
a_{1} & =a_{0}|\mathbf{P}|+b_{0}\{1-|\mathbf{P}|\}+\operatorname{Tr}\left(\mathbf{q} \overline{\mathcal{C}}_{0} \overline{\mathbf{P}}\right) \\
& =a_{0}+\left(b_{0}-a_{0}\right)\{1-|\mathbf{P}|\}+\operatorname{Tr}\left(\mathbf{q} \overline{\mathcal{C}}_{0} \overline{\mathbf{P}}\right) .
\end{aligned}
$$

Then since the sum (360) is conserved we have

$$
b_{1}=a_{0}+b_{0}-a_{1} \text {. }
$$

So to obtain $a_{1}$ and $b_{1}$ we need to compute $|\mathbf{P}|$ and $\operatorname{Tr}\left(\mathbf{q} \overline{\mathcal{C}}_{0} \overline{\mathbf{P}}\right)$. 
Now it follows from equations (353-354) and (356) that matrices $\mathbf{P}, \mathbf{q}, \mathbf{p}$, $\mathbf{Q}$ and $\mathcal{C}_{0}$ are of the form

$$
\begin{aligned}
& \mathbf{P}=\left(\begin{array}{rr}
P_{11} & P_{12} \\
-P_{12} & P_{11}
\end{array}\right), \quad \mathbf{q}=\left(\begin{array}{rr}
q_{11} & q_{12} \\
-q_{12} & q_{11}
\end{array}\right) \\
& \mathbf{p}=\left(\begin{array}{rr}
p_{11} & p_{12} \\
-p_{12} & p_{11}
\end{array}\right), \quad \mathbf{Q}=\left(\begin{array}{rr}
Q_{11} & Q_{12} \\
-Q_{12} & Q_{11}
\end{array}\right)
\end{aligned}
$$

and

$$
\mathcal{C}_{0}=\left(\begin{array}{rr}
c_{0} & h_{0} \\
-h_{0} & c_{0}
\end{array}\right) \text {. }
$$

Thus

$$
\begin{gathered}
|\mathbf{P}|=P_{11}^{2}+P_{12}^{2} \\
\mathbf{q} \overline{\mathcal{C}}_{0} \overline{\mathbf{P}}=\left(\begin{array}{rr}
q_{11} & q_{12} \\
-q_{12} & q_{11}
\end{array}\right)\left(\begin{array}{rr}
c_{0} & -h_{0} \\
h_{0} & c_{0}
\end{array}\right)\left(\begin{array}{rr}
P_{11} & -P_{12} \\
P_{12} & P_{11}
\end{array}\right) \\
\mathbf{q} \overline{\mathcal{C}}_{0} \overline{\mathbf{P}}=\left(\begin{array}{rr}
q_{11} c_{0}+q_{12} h_{0} & q_{12} c_{0}-q_{11} h_{0} \\
q_{11} h_{0}-q_{12} c_{0} & q_{11} c_{0}+q_{12} h_{0}
\end{array}\right)\left(\begin{array}{rr}
P_{11} & -P_{12} \\
P_{12} & P_{11}
\end{array}\right)
\end{gathered}
$$

and

$$
\operatorname{Tr}\left(\mathbf{q} \overline{\mathcal{C}}_{0} \overline{\mathbf{P}}\right)=2\left\{\left(q_{11} c_{0}+q_{12} h_{0}\right) P_{11}+\left(q_{12} c_{0}-q_{11} h_{0}\right) P_{12}\right\}
$$

For the computation of

$$
\mathcal{C}_{1}=\left(\begin{array}{rr}
c_{1} & h_{1} \\
-h_{1} & c_{1}
\end{array}\right)
$$

we have

$$
c_{1}=a_{0}(\mathbf{P} \overline{\mathbf{p}})_{11}+b_{0}(\mathbf{q} \overline{\mathbf{Q}})_{11}+\left(\mathbf{q} \overline{\mathcal{C}}_{0} \overline{\mathbf{p}}\right)_{11}+\left(\mathbf{P} \mathcal{C}_{0} \overline{\mathbf{Q}}\right)_{11}
$$

and

$$
h_{1}=a_{0}(\mathbf{P} \overline{\mathbf{p}})_{12}+b_{0}(\mathbf{q} \overline{\mathbf{Q}})_{12}+\left(\mathbf{q} \overline{\mathcal{C}}_{0} \overline{\mathbf{p}}\right)_{12}+\left(\mathbf{P} \mathcal{C}_{0} \overline{\mathbf{Q}}\right)_{12}
$$

where

$$
\begin{aligned}
\mathbf{P} \overline{\mathbf{p}} & =\left(\begin{array}{rr}
P_{11} & P_{12} \\
-P_{12} & P_{11}
\end{array}\right)\left(\begin{array}{rr}
p_{11} & -p_{12} \\
p_{12} & p_{11}
\end{array}\right) \\
\mathbf{q} \overline{\mathbf{Q}} & =\left(\begin{array}{rr}
q_{11} & q_{12} \\
-q_{12} & q_{11}
\end{array}\right)\left(\begin{array}{rr}
Q_{11} & -Q_{12} \\
Q_{12} & Q_{11}
\end{array}\right)
\end{aligned}
$$




$$
\begin{aligned}
& (\mathbf{P} \overline{\mathbf{p}})_{11}=P_{11} p_{11}+P_{12} p_{12}, \quad(\mathbf{P} \overline{\mathbf{p}})_{12}=P_{12} p_{11}-P_{11} p_{12} \\
& (\mathbf{q} \overline{\mathbf{Q}})_{11}=q_{11} Q_{11}+q_{12} Q_{12}, \quad(\mathbf{q} \overline{\mathbf{Q}})_{12}=q_{12} Q_{11}-q_{11} Q_{12} \\
& \mathbf{q} \overline{\mathcal{C}}_{0} \overline{\mathbf{p}}=\left(\begin{array}{rr}
q_{11} & q_{12} \\
-q_{12} & q_{11}
\end{array}\right)\left(\begin{array}{rr}
c_{0} & -h_{0} \\
h_{0} & c_{0}
\end{array}\right)\left(\begin{array}{rr}
p_{11} & -p_{12} \\
p_{12} & p_{11}
\end{array}\right) \\
& \mathbf{q} \overline{\mathcal{C}}_{0} \overline{\mathbf{p}}=\left(\begin{array}{ll}
q_{11} c_{0}+q_{12} h_{0} & q_{12} c_{0}-q_{11} h_{0} \\
q_{11} h_{0}-q_{12} c_{0} & q_{11} c_{0}+q_{12} h_{0}
\end{array}\right)\left(\begin{array}{rr}
p_{11} & -p_{12} \\
p_{12} & p_{11}
\end{array}\right) \\
& \left(\mathbf{q} \overline{\mathcal{C}}_{0} \overline{\mathbf{p}}\right)_{11}=\left(q_{11} c_{0}+q_{12} h_{0}\right) p_{11}+\left(q_{12} c_{0}-q_{11} h_{0}\right) p_{12} \\
& \left(\mathbf{q} \overline{\mathcal{C}}_{0} \overline{\mathbf{p}}\right)_{12}=\left(q_{12} c_{0}-q_{11} h_{0}\right) p_{11}-\left(q_{11} c_{0}+q_{12} h_{0}\right) p_{12} \\
& \mathbf{P} \mathcal{C}_{0} \overline{\mathbf{Q}}=\left(\begin{array}{rr}
P_{11} & P_{12} \\
-P_{12} & P_{11}
\end{array}\right)\left(\begin{array}{rr}
c_{0} & h_{0} \\
-h_{0} & c_{0}
\end{array}\right)\left(\begin{array}{rr}
Q_{11} & -Q_{12} \\
Q_{12} & Q_{11}
\end{array}\right) \\
& \mathbf{P} \mathcal{C}_{0} \overline{\mathbf{Q}}=\left(\begin{array}{cc}
P_{11} c_{0}-P_{12} h_{0} & P_{11} h_{0}+P_{12} c_{0} \\
-P_{12} c_{0}-P_{11} h_{0} & P_{11} c_{0}-P_{12} h_{0}
\end{array}\right)\left(\begin{array}{rr}
Q_{11} & -Q_{12} \\
Q_{12} & Q_{11}
\end{array}\right) \\
& \left(\mathbf{P} \mathcal{C}_{0} \overline{\mathbf{Q}}\right)_{11}=\left(P_{11} c_{0}-P_{12} h_{0}\right) Q_{11}+\left(P_{11} h_{0}+P_{12} c_{0}\right) Q_{12}
\end{aligned}
$$

and

$$
\left(\mathbf{P} \mathcal{C}_{0} \overline{\mathbf{Q}}\right)_{12}=\left(P_{11} h_{0}+P_{12} c_{0}\right) Q_{11}-\left(P_{11} c_{0}-P_{12} h_{0}\right) Q_{12}
$$

\section{Appendix $\mathrm{C}$}

Consider the case in which

$$
\mathbf{P}=d_{0} d \mathcal{A}+e_{0} e \mathcal{B}
$$

and

$$
\mathcal{C}_{0}=\mathbf{0}
$$


where

$$
\mathcal{A}=\left(\begin{array}{rr}
\cos \psi_{1} & \sin \psi_{1} \\
-\sin \psi_{1} & \cos \psi_{1}
\end{array}\right), \quad \mathcal{B}=\left(\begin{array}{rr}
\cos \psi_{2} & \sin \psi_{2} \\
-\sin \psi_{2} & \cos \psi_{2}
\end{array}\right)
$$

and

$$
d_{0}^{2}+e_{0}^{2}=1, \quad d^{2}+e^{2}=1
$$

As shown in Appendix A we then have

$$
a_{1}=a_{0}+\left(b_{0}-a_{0}\right)\{1-|\mathbf{P}|\}
$$

and

$$
b_{1}=b_{0}-\left(b_{0}-a_{0}\right)\{1-|\mathbf{P}|\}
$$

Here

$$
|\mathbf{P}|=P_{11}^{2}+P_{12}^{2}
$$

where

$$
P_{11}=d_{0} d \cos \psi_{1}+e_{0} e \cos \psi_{2}
$$

and

$$
P_{12}=d_{0} d \sin \psi_{1}+e_{0} e \sin \psi_{2}
$$

Thus

$$
|\mathbf{P}|=d_{0}^{2} d^{2}+e_{0}^{2} e^{2}+2 d_{0} e_{0} d e\left\{\cos \psi_{1} \cos \psi_{2}+\sin \psi_{1} \sin \psi_{2}\right\}
$$

and using

$$
\begin{gathered}
\cos \psi_{1} \cos \psi_{2}+\sin \psi_{1} \sin \psi_{2}=\cos \left(\psi_{1}-\psi_{2}\right) \\
d_{0}^{2} d^{2}+e_{0}^{2} e^{2}=d_{0}^{2}\left(1-e^{2}\right)+e_{0}^{2}\left(1-d^{2}\right) \\
d_{0}^{2} d^{2}+e_{0}^{2} e^{2}=1-\left\{d_{0}^{2} e^{2}+e_{0}^{2} d^{2}\right\}
\end{gathered}
$$

we have

$$
|\mathbf{P}|=1-\left\{d_{0}^{2} e^{2}+e_{0}^{2} d^{2}\right\}+2 d_{0} e_{0} d e \cos \left(\psi_{1}-\psi_{2}\right)
$$

and

$$
1-|\mathbf{P}|=d_{0}^{2} e^{2}+e_{0}^{2} d^{2}-2 d_{0} e_{0} d e \cos \left(\psi_{1}-\psi_{2}\right) .
$$

Using this in (402) and (403) then gives

$$
a_{1}=F-2\left(b_{0}-a_{0}\right) d_{0} e_{0} d e \cos \left(\psi_{1}-\psi_{2}\right)
$$

and

$$
b_{1}=G+2\left(b_{0}-a_{0}\right) d_{0} e_{0} d e \cos \left(\psi_{1}-\psi_{2}\right)
$$


where

$$
\begin{aligned}
& F=a_{0}+\left(b_{0}-a_{0}\right)\left\{d_{0}^{2} e^{2}+e_{0}^{2} d^{2}\right\} \\
& G=b_{0}-\left(b_{0}-a_{0}\right)\left\{d_{0}^{2} e^{2}+e_{0}^{2} d^{2}\right\}
\end{aligned}
$$

Consider now the case in which

$$
\mathbf{P}=d_{0} e \mathcal{A}+e_{0} d \mathcal{B}
$$

and

$$
\mathcal{C}_{0}=\mathbf{0}
$$

We then have

$$
\begin{gathered}
a_{1}=a_{0}+\left(b_{0}-a_{0}\right)\{1-|\mathbf{P}|\} \\
b_{1}=b_{0}-\left(b_{0}-a_{0}\right)\{1-|\mathbf{P}|\} \\
P_{11}=d_{0} e \cos \psi_{1}+e_{0} d \cos \psi_{2} \\
P_{12}=d_{0} e \sin \psi_{1}+e_{0} d \sin \psi_{2} \\
|\mathbf{P}|=d_{0}^{2} e^{2}+e_{0}^{2} d^{2}+2 d_{0} e_{0} d e\left\{\cos \psi_{1} \cos \psi_{2}+\sin \psi_{1} \sin \psi_{2}\right\}
\end{gathered}
$$

and

$$
1-|\mathbf{P}|=1-\left\{d_{0}^{2} e^{2}+e_{0}^{2} d^{2}\right\}-2 d_{0} e_{0} d e \cos \left(\psi_{1}-\psi_{2}\right)
$$

Thus

$$
a_{1}=G-2\left(b_{0}-a_{0}\right) d_{0} e_{0} d e \cos \left(\psi_{1}-\psi_{2}\right)
$$

and

$$
b_{1}=F+2\left(b_{0}-a_{0}\right) d_{0} e_{0} d e \cos \left(\psi_{1}-\psi_{2}\right)
$$

where

$$
\begin{aligned}
& G=b_{0}-\left(b_{0}-a_{0}\right)\left\{d_{0}^{2} e^{2}+e_{0}^{2} d^{2}\right\} \\
& F=a_{0}+\left(b_{0}-a_{0}\right)\left\{d_{0}^{2} e^{2}+e_{0}^{2} d^{2}\right\}
\end{aligned}
$$

\section{References}

[1] G. Guignard, "The General Theory of All Sum and Difference Resonances in a Three-Dimensional Magnetic Field in a Synchrotron", CERN Report 76-06 (1976).

[2] G. Guignard, "Betatron Coupling and the Related Impact of Radiation", Phys. Rev. E 51, 6104 (1995) 
[3] D. A. Edwards and L. C. Teng, "Parameterization of Linear Coupled Motion in Periodic Systems", IEEE Trans. on Nucl. Sc. 20, 885 (1973). L. C. Teng, "Concerning n-Dimensional Coupled Motions", NAL Report FN-229, May 3, 1971.

[4] M. Billing, "The Theory of Weakly Coupled Transverse Motion in Storage Rings", Cornell University Report CBN 85-2 (1985).

[5] T. Roser, "Generalized Treatment of Linear Motion in Two-Dimensional Periodic Systems", University of Michigan Report UM-HE 88-8 (1988).

[6] D. Sagan and D. Rubin, "Linear Analysis of Coupled Lattices", Phys. Rev. ST Accel. and Beams 2, 074001 (1999)

[7] K. L. Brown and R. V. Servranckx, Particle Accelerators 36, 121-139 (1991).

[8] E. D. Courant and H. S. Snyder, Annals of Physics 3, 1-48 (1958).

[9] A.J. Dragt, "Lectures on Nonlinear Orbit Dynamics", Physics of High Energy Particle Accelerators, AIP Conference Proceedings 87, 147-313 (1982).

[10] F. Christoph Iselin, "The MAD Program Physical Methods Manual", CERN, Geneva, September 15, 1994.

[11] Yun Luo, "Linear Coupling Parametrization in the Action-Angle Frame", Phys. Rev. ST Accel. and Beams 7, 124001 (2004)

[12] C. Carli, et al, "Emittance Exchange by Crossing a Coupling Resonance", Proceedings of EPAC 2002, Paris, France, p. 1157.

[13] L.D. Landau and E.M. Lifshitz, "Mechanics", Third Edition, Pergamon Press, 1976, pp. 154-157.

[14] R. Calaga, R. Tomas and A. Franchi, "Betatron Coupling: Merging Hamiltonian and Matrix Approaches", Phys. Rev. ST Accel. and Beams 8, 034001 (2005) 


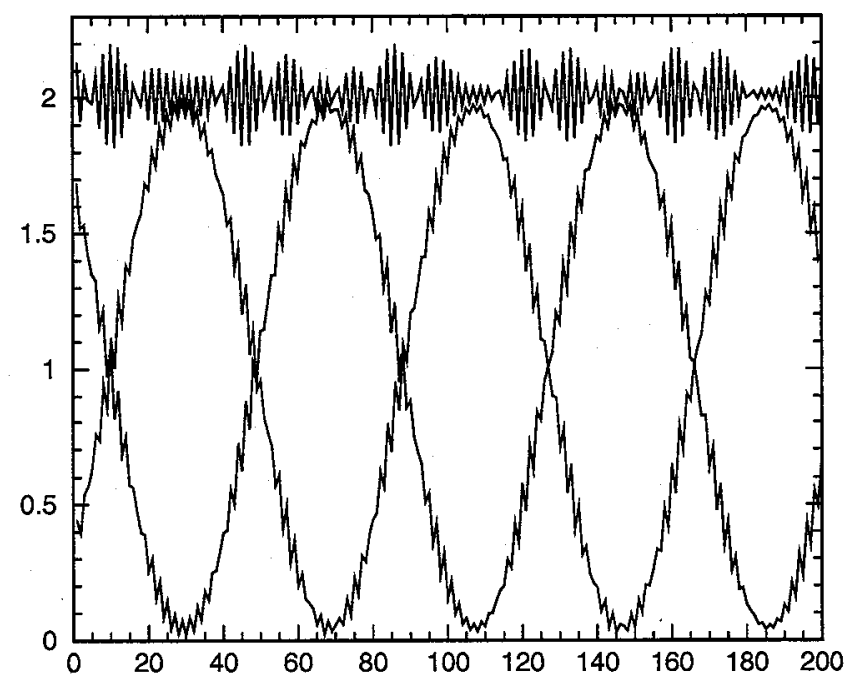

Figure 1: Horizontal and vertical amplitudes $J_{x}$ and $J_{y}$ and their sum versus turn plotted in black, red and blue respectively. Here normalized coupling matrix parameters $A=0.6$ and $B=0.06$. The small-amplitude highfrequency oscillations seen on all of the curves are due to the parameter $B$ being small but nonzero.

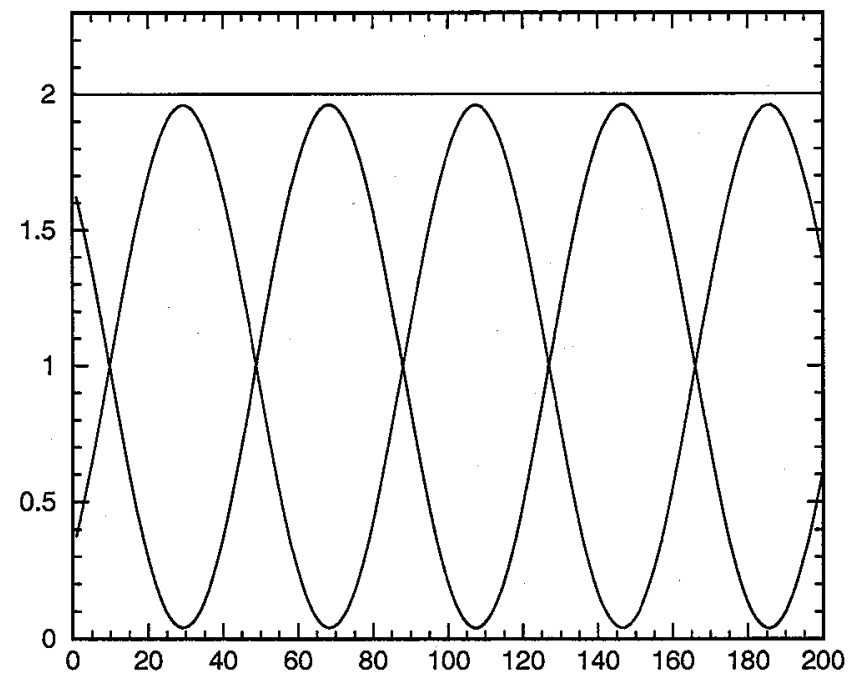

Figure 2: Amplitudes $J_{x}$ and $J_{y}$ and their sum obtained with normalized coupling matrix parameters $A=0.6$ and $B=0$. Here the high-frequency oscillations are gone and the sum is constant. 


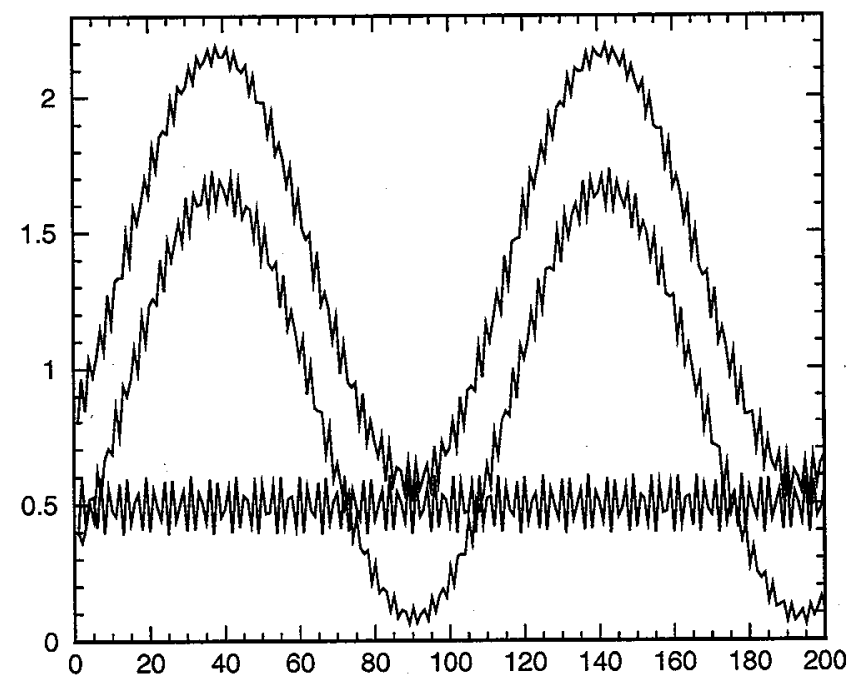

Figure 3: Amplitudes $J_{x}$ and $J_{y}$ and their difference plotted in black, red and green respectively. Here normalized coupling matrix parameters $A=0.05$ and $B=0.5$. The small-amplitude high-frequency oscillations seen on all of the curves are due to the parameter $A$ being small but nonzero.

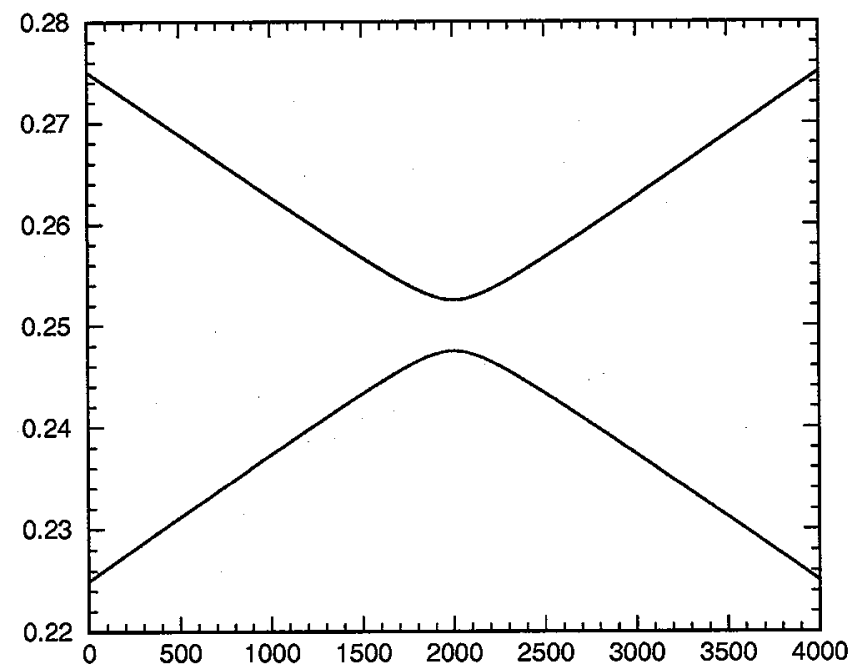

Figure 4: Normal-mode tunes $Q_{1}$ and $Q_{2}$ versus turn number $n$. Here the black curve is $Q_{1 n}$ and the red curve is $Q_{2 n}$. These tunes are given by equations (270-274) with $Q=0.25, \Delta Q_{\max }=0.05, \Delta Q_{\min }=0.005$ and $2 N=4000$. 


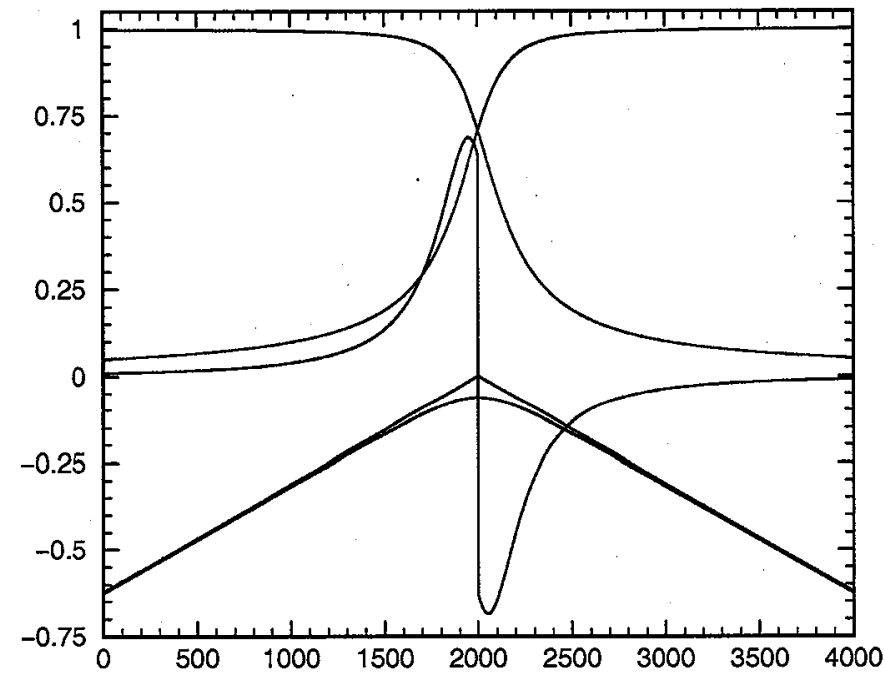

Figure 5: Parameters $U_{n}, T_{n}, d_{n}, e_{n}$ and $\xi_{n}$ plotted in black, red, blue, green and violet, respectively, for the tunes shown in Figure 4. Note that $T_{n}$ reaches zero at $n=N=2000$ but does not pass through zero.

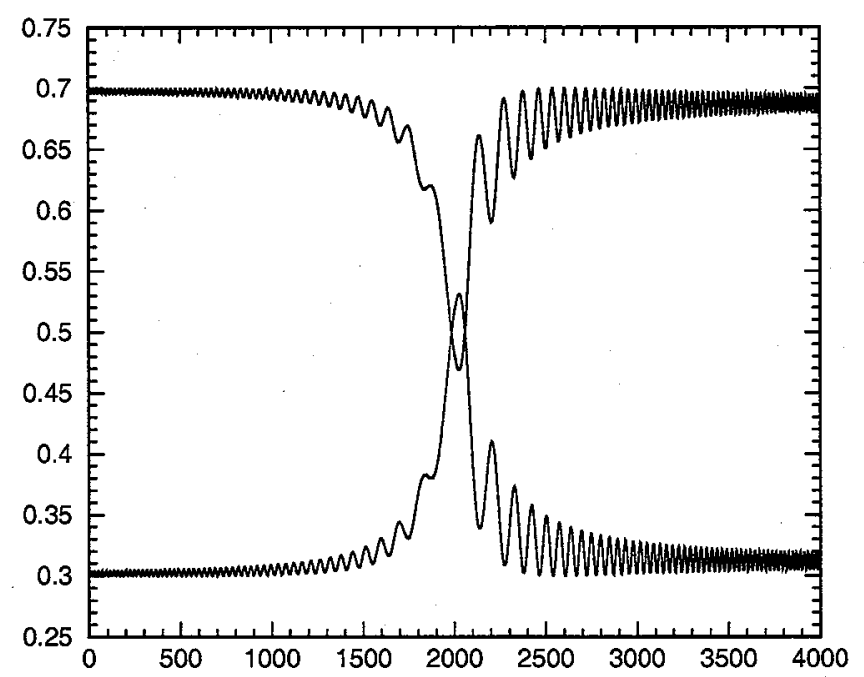

Figure 6: Exact horizontal and vertical emittances $\epsilon_{x n}$ and $\epsilon_{y n}$ plotted in black and red, respectively, for the tunes and parameters of Figures 4 and 5 . These were obtained by iteration of equation (201) with starting parameters $\epsilon_{x 0}=0.7, \epsilon_{y 0}=0.3, c_{0}=0$ and $h_{0}=0$. The emittances are not exchanged in this case. 


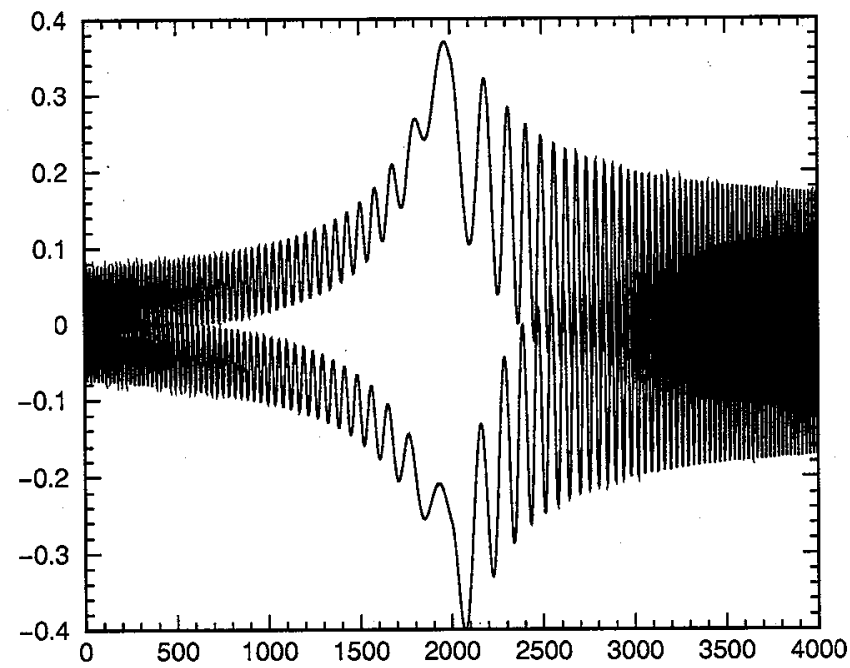

Figure 7: Elements $c_{n}$ and $h_{n}$ of normalized beam ellipsoid matrix $\mathcal{E}_{n}$ plotted in green and blue, respectively, for the tunes and parameters of Figures 4 and 5 .

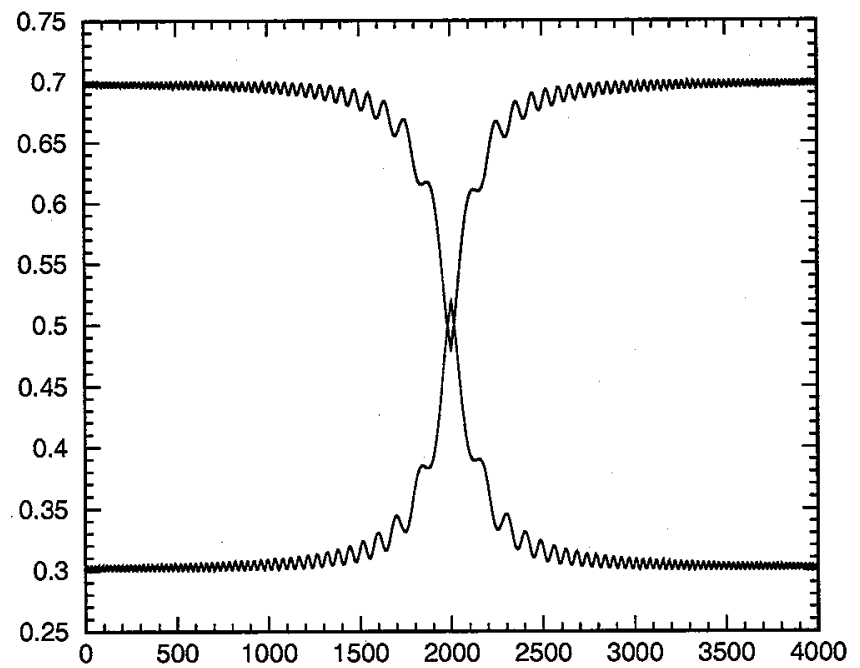

Figure 8: Approximate emittances $\epsilon_{x n}$ and $\epsilon_{y n}$ plotted in black and red, respectively, for the tunes and parameters of Figures 4 and 5 . These were obtained from equations (253-256). 


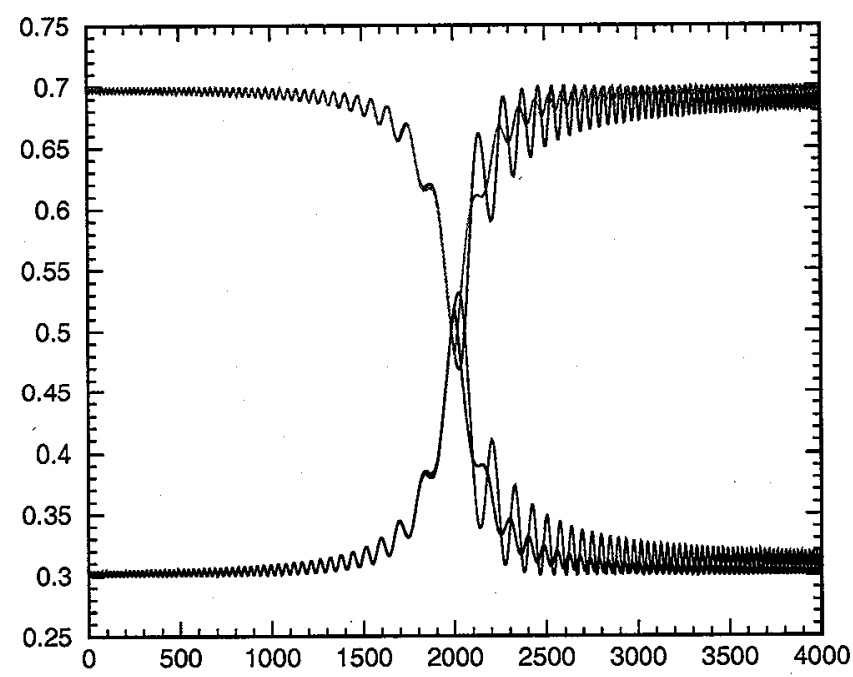

Figure 9: Overlay of approximate on exact emittances from Figures 6 and 8. The approximate emittances are shown in gold and blue. Note that there is good agreement up to turn 2000 .

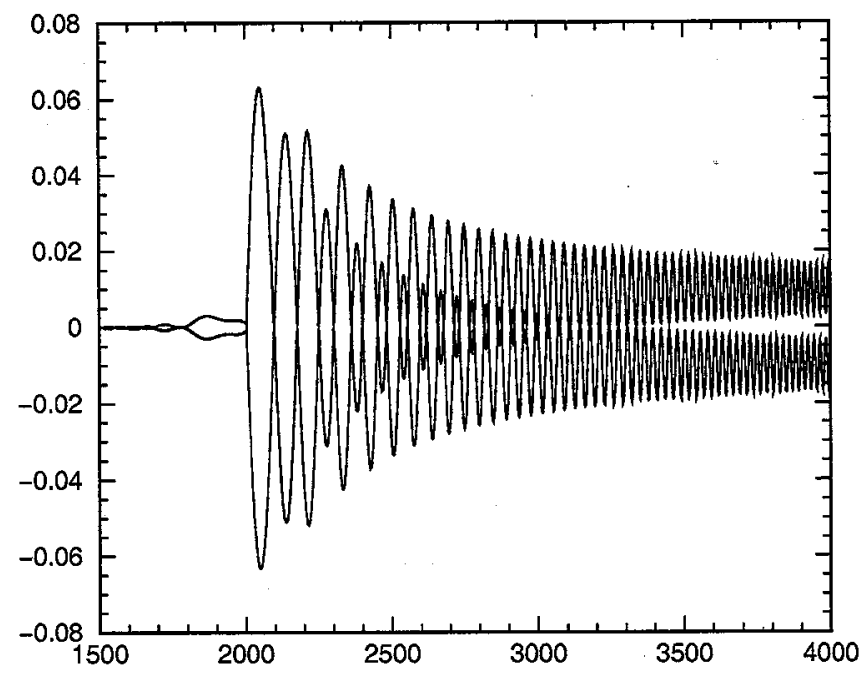

Figure 10: Difference between exact and approximate emittances from Figure 9. The horizontal and vertical differences (approximate minus exact) are shown in black and red respectively. 


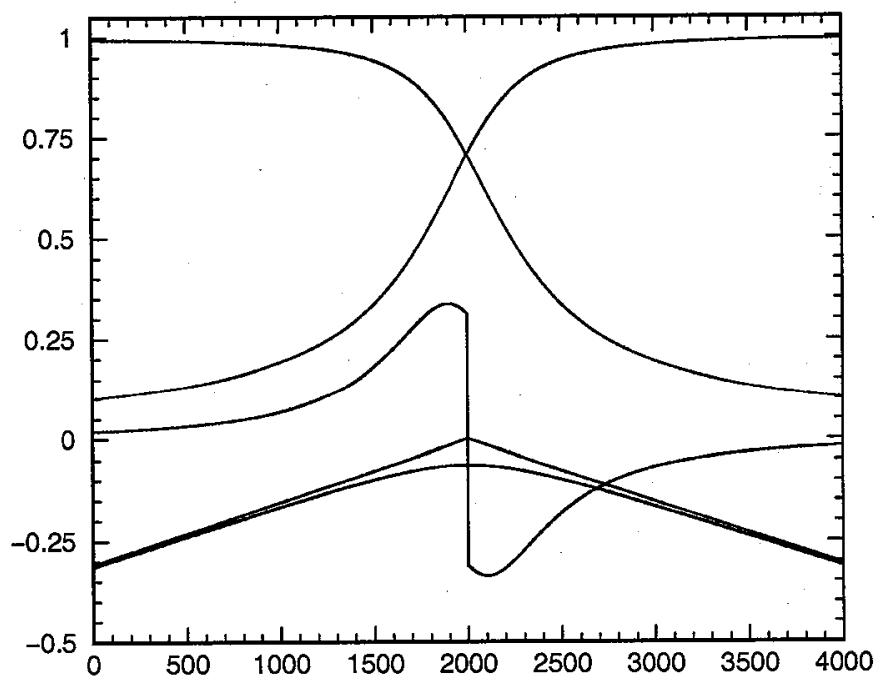

Figure 11: Parameters $U_{n}, T_{n}, d_{n}, e_{n}$ and $\xi_{n}$ plotted in black, red, blue, green and violet, respectively, for the case in which $Q_{\max }$ is reduced from 0.05 to 0.025 . Comparing with Figure 5 we see that the adiabatic parameter $\xi_{n}$ is reduced by a factor of 2 .

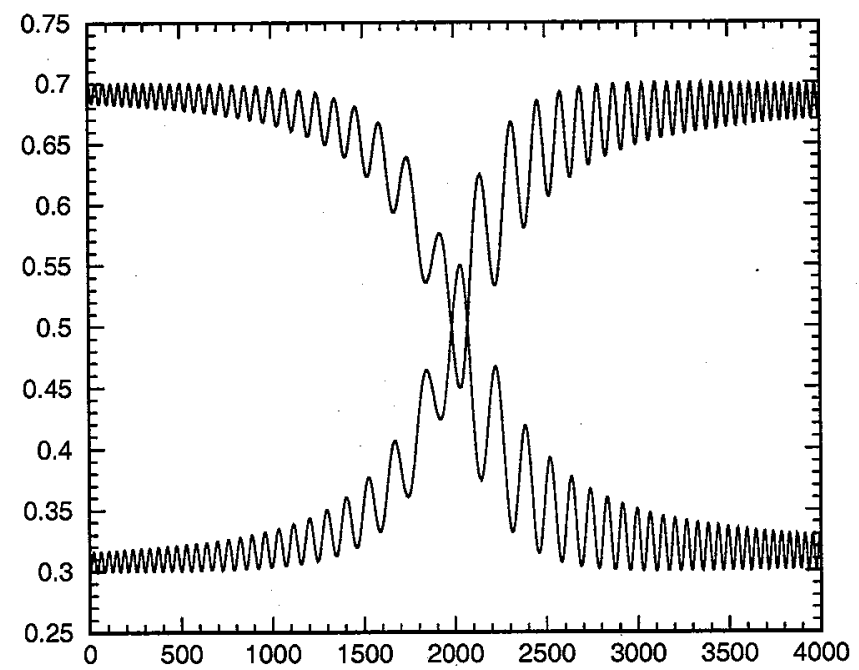

Figure 12: Exact emittances $\epsilon_{x n}$ and $\epsilon_{y n}$ plotted in black and red, respectively, for the case in which $Q_{\max }$ is reduced from 0.05 to 0.025 . 


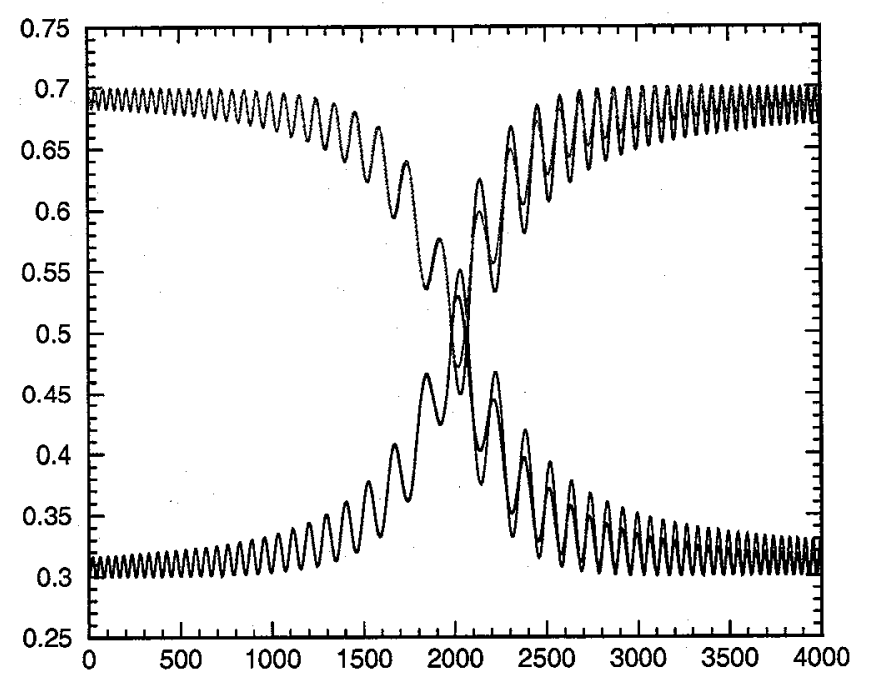

Figure 13: Overlay of approximate on exact emittances for the case in which $Q_{\max }$ is reduced from 0.05 to 0.025 . The approximate emittances are shown in gold and blue. Note the improved agreement between approximate and exact emittances.

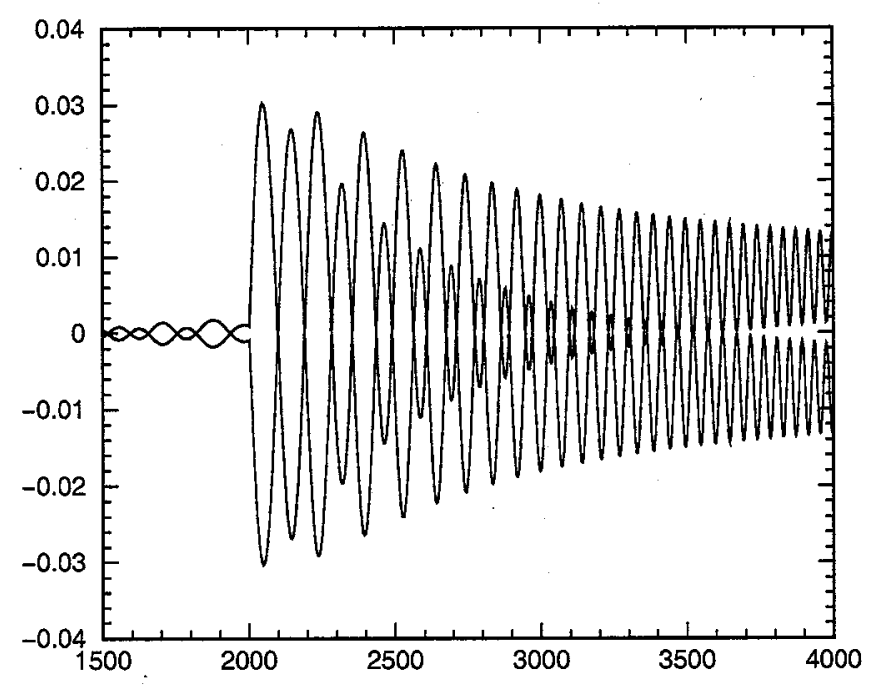

Figure 14: Difference between exact and approximate emittances for the case in which $Q_{\max }$ is reduced from 0.05 to 0.025 . Comparing with Figure 10 we see that the differences have been reduced by a factor of 2 . 


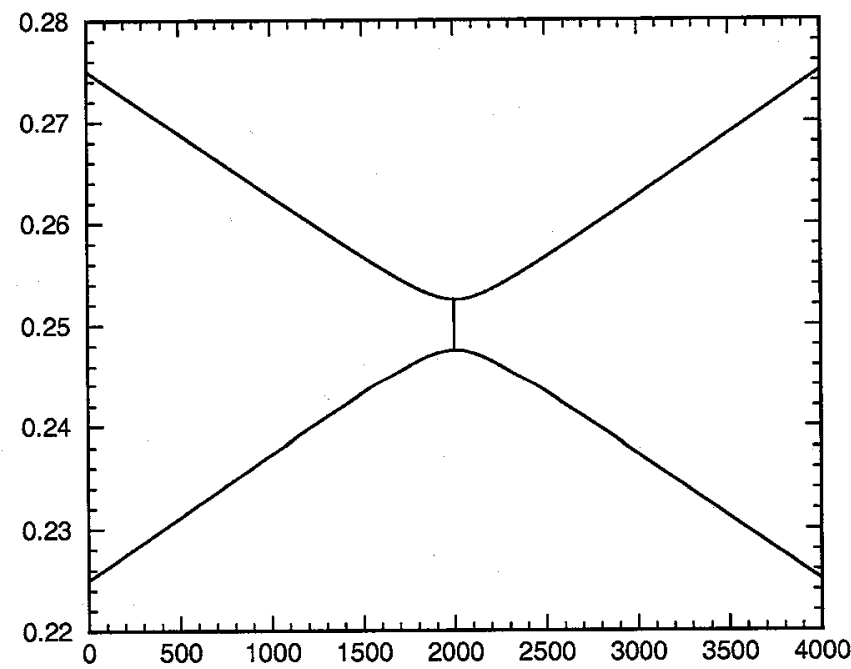

Figure 15: Normal-mode tunes $Q_{1 n}$ and $Q_{2 n}$ plotted in black and red respectively. These tunes are given by equations (270-272) and (287-288) with $Q=0.25, \Delta Q_{\max }=0.05, \Delta Q_{\min }=0.005$ and $2 N=4000$.

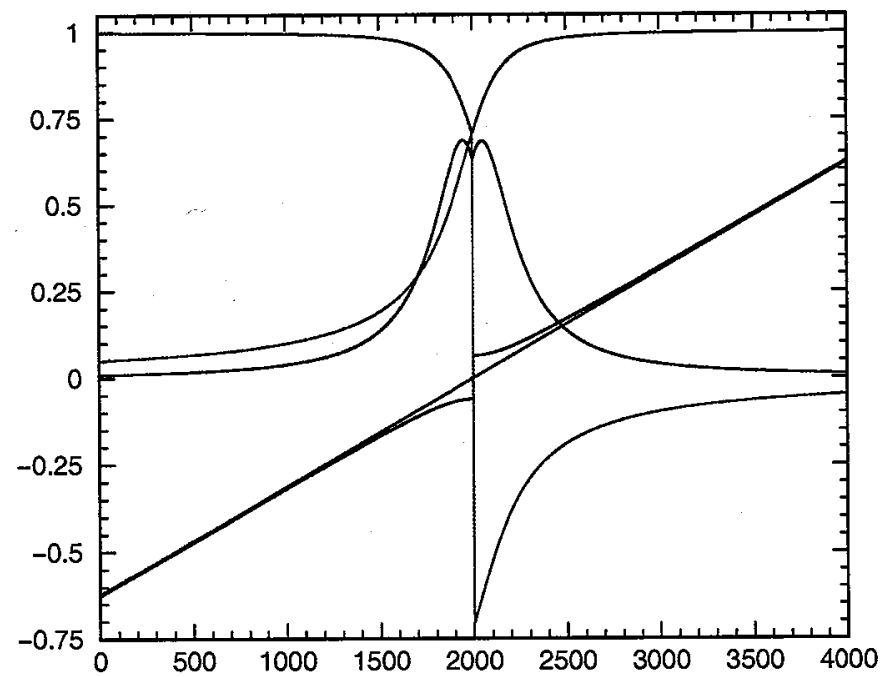

Figure 16: Parameters $U_{n}, T_{n}, d_{n}, e_{n}$ and $\xi_{n}$ plotted in black, red, blue, green and violet, respectively, for the tunes shown in Figure 15. Note that $T_{n}$ is monotonically increasing and passes through zero at $n=N=2000$. 


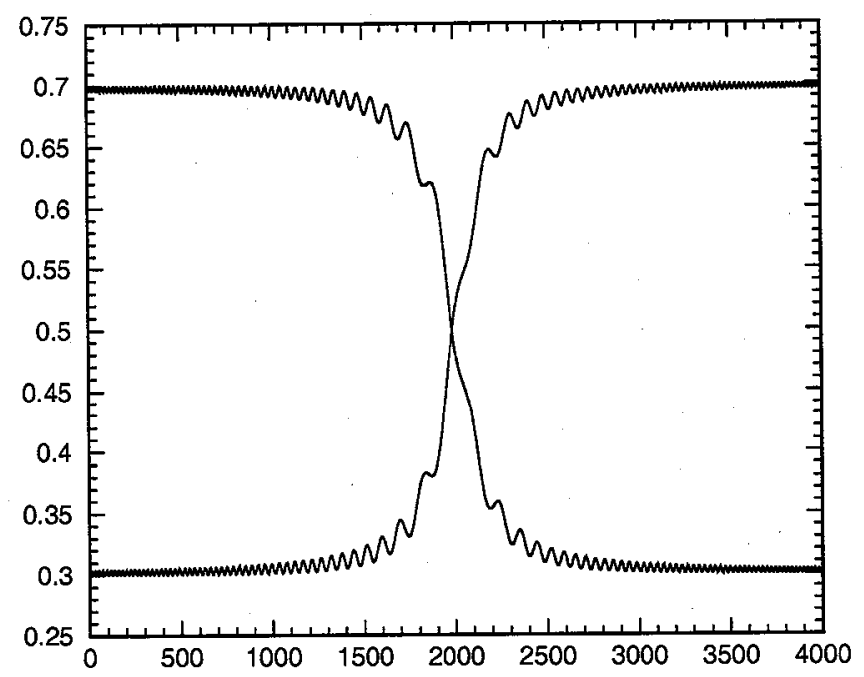

Figure 17: Exact horizontal and vertical emittances $\epsilon_{x n}$ and $\epsilon_{y n}$ plotted in black and red, respectively, for the tunes and parameters of Figures 15 and 16. These were obtained by iteration of equations (201) and (292) with starting parameters $\epsilon_{x 0}=0.7, \epsilon_{y 0}=0.3, c_{0}=0$ and $h_{0}=0$. The emittances are exchanged in this case.

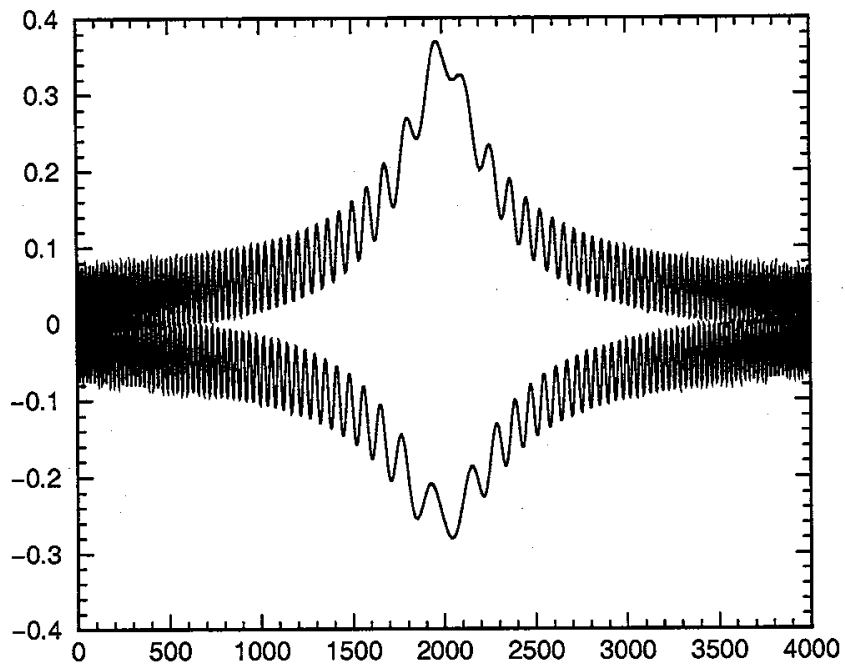

Figure 18: Elements $c_{n}$ and $h_{n}$ of normalized beam ellipsoid matrix $\mathcal{E}_{n}$ plotted in green and blue, respectively, for the tunes and parameters of Figures 15 and 16. 


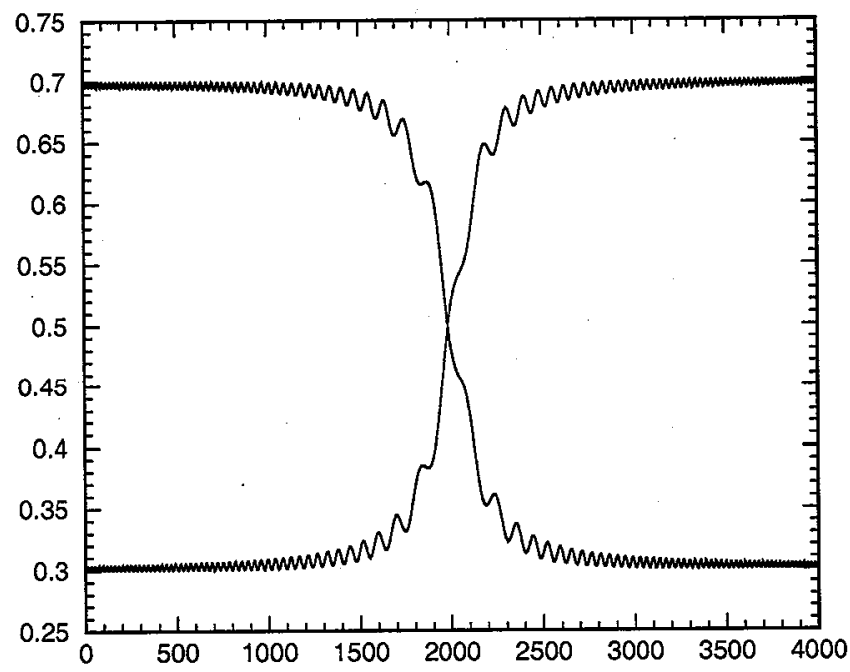

Figure 19: Approximate emittances $\epsilon_{x n}$ and $\epsilon_{y n}$ plotted in black and red, respectively, for the tunes and parameters of Figures 15 and 16. These were obtained from equations (253-256) and (338-339).

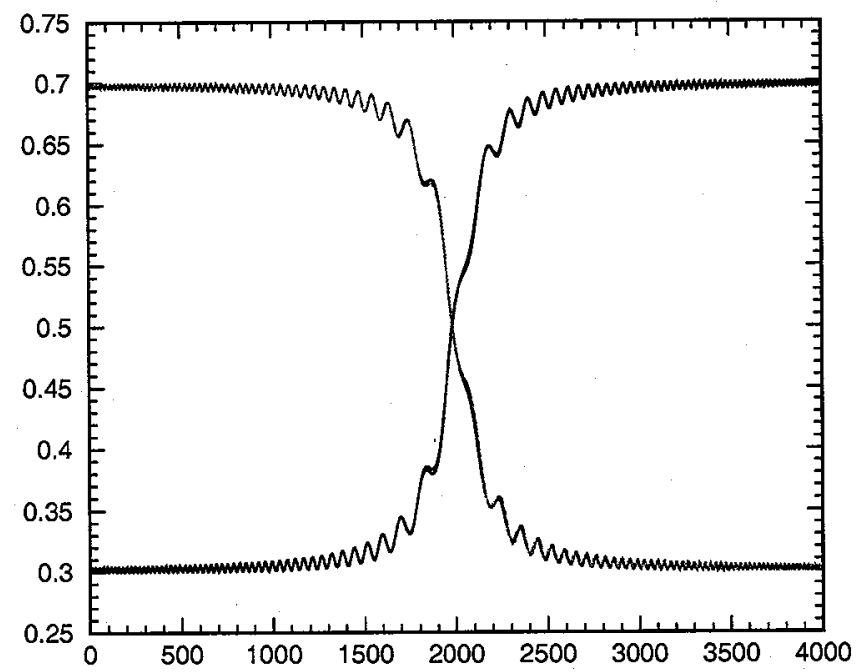

Figure 20: Overlay of approximate on exact emittances from Figures 17 and 19. The approximate emittances are shown in gold and blue. Note that there is good agreement over the whole range of 4000 turns. 


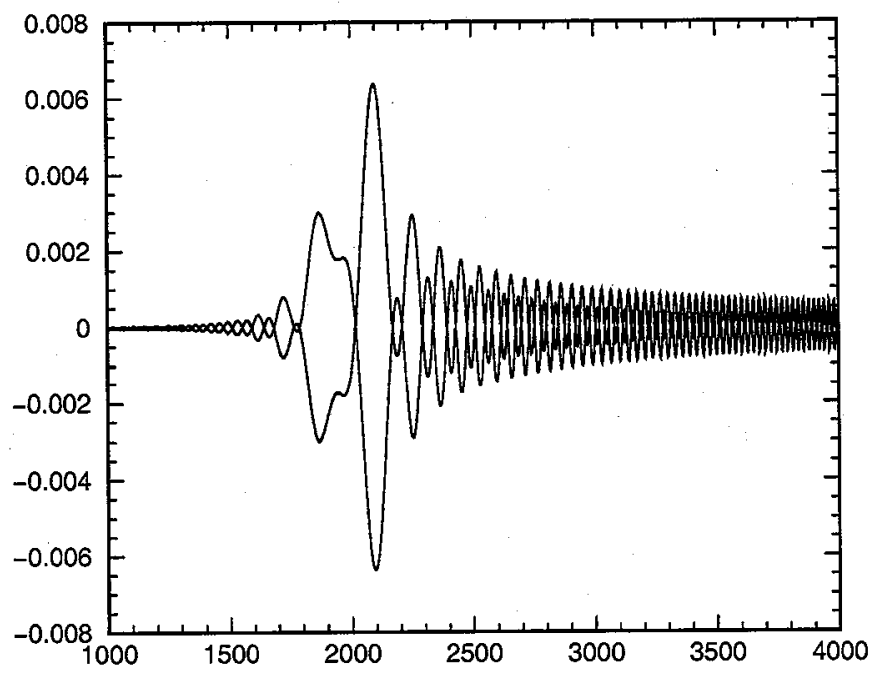

Figure 21: Difference between exact and approximate emittances from Figure 20. The horizontal and vertical differences (approximate minus exact) are shown in black and red respectively.

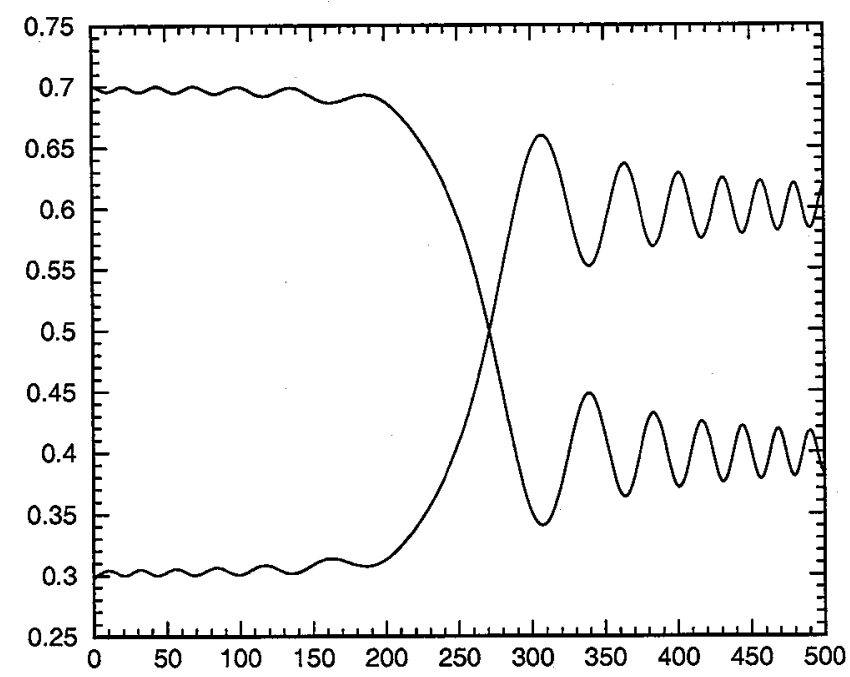

Figure 22: Exact horizontal and vertical emittances $\epsilon_{x n}$ and $\epsilon_{y n}$ plotted in black and red, respectively, for the same parameters as before but with $2 N=500$ turns. 


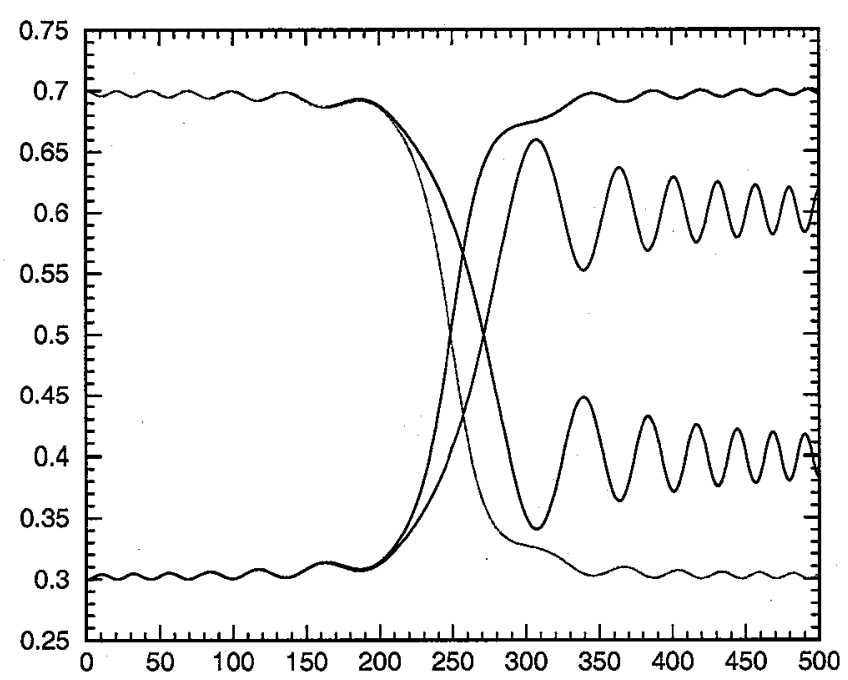

Figure 23: Overlay of approximate on exact emittances from Figure 22. The approximate emittances are shown in gold and blue. Note that there is good agreement up to about 200 turns; afterward the agreement is poor.

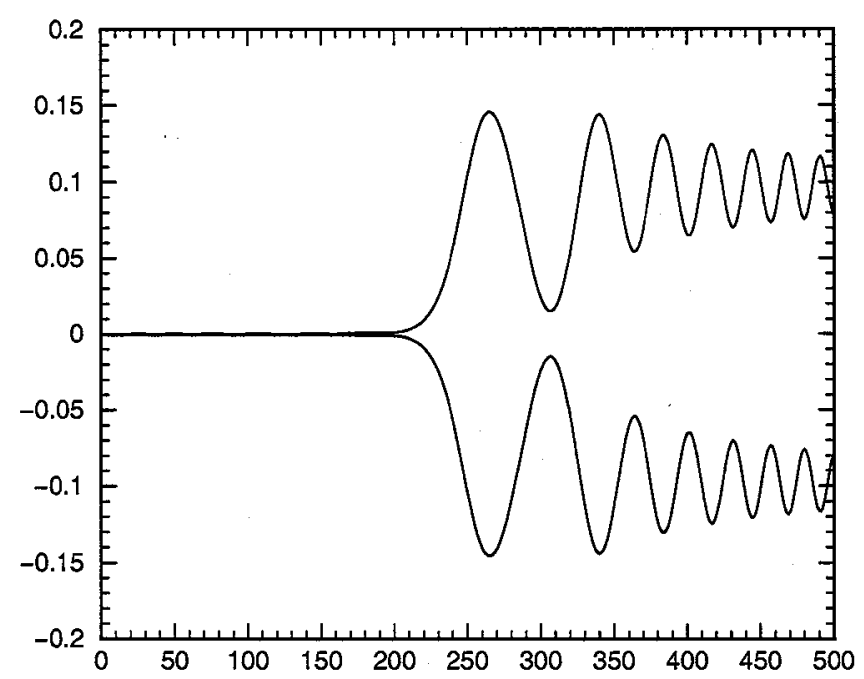

Figure 24: Difference between exact and approximate emittances from Figure 23. The horizontal and vertical differences (approximate minus exact) are shown in black and red respectively. 